\title{
A Strongly Degenerate Quasilinear Equation: the Elliptic Case
}

\author{
FUENSANTA ANDREU - VICENT CASELLES - JOSÉ M. MAZÓN
}

\begin{abstract}
We prove existence and uniqueness of entropy solutions for the Neumann problem for the quasilinear elliptic equation $u-\operatorname{div} \mathbf{a}(u, D u)=v$, where $v \in L^{1}, \mathbf{a}(z, \xi)=\nabla_{\xi} f(z, \xi)$, and $f$ is a convex function of $\xi$ with linear growth as $\|\xi\| \rightarrow \infty$, satisfying other additional assumptions. In particular, this class includes the case where $f(z, \xi)=\varphi(z) \psi(\xi), \varphi>0, \psi$ being a convex function with linear growth as $\|\xi\| \rightarrow \infty$. In the second part of this work, using Crandall-Ligget's iteration scheme, this result will permit us to prove existence and uniqueness of entropy solutions for the corresponding parabolic problem with initial data in $L^{1}$.
\end{abstract}

Mathematics Subject Classification (2000): $35 \mathrm{~J} 60$ (primary); 47H06, 47H20 (secondary).

\section{1. - Introduction}

Let $\Omega$ be a bounded set in $\mathbb{R}^{N}$ with boundary $\partial \Omega$ of class $C^{1}$. We are interested in the problem

$$
\begin{cases}u-\operatorname{div} \mathbf{a}(u, D u)=v & \text { in } \Omega \\ \frac{\partial u}{\partial \eta}=0 & \text { on } \partial \Omega,\end{cases}
$$

where $v \in L^{1}(\Omega), \mathbf{a}(z, \xi)=\nabla_{\xi} f(z, \xi), f$ being a function with linear growth as $\|\xi\| \rightarrow \infty$ and $\frac{\partial}{\partial \eta}$ is the Neumann boundary operator associated to $\mathbf{a}(u, D u)$, i.e.,

$$
\frac{\partial u}{\partial \eta}:=\mathbf{a}(u, D u) \cdot v,
$$

with $v$ the unit outward normal on $\partial \Omega$.

Our purpose in this paper is to define a notion of entropy solution for (1.1), and prove existence and uniqueness results when the right hand side $v$ is in $L^{1}(\Omega)$ (or in $L^{\infty}(\Omega)$, depending on our set of assumptions). Besides the fact that the elliptic problem is interesting by itself, this result permits us to 
associate to the expression $-\operatorname{div} \mathbf{a}(u, D u)$ with Neumann boundary condition an $m$-accretive operator $\mathcal{B}$ in $L^{1}(\Omega)$ with dense domain, thus, generating a non-linear contraction semigroup $T(t)$ in $L^{1}(\Omega)$ ([11], [18], [19]), and to use Crandall-Ligget's iteration scheme to define the function

$$
u(t):=T(t) u_{0}=\lim _{n \rightarrow \infty}\left(I+\frac{t}{n} \mathcal{B}\right)^{-n} u_{0}, \quad u_{0} \in L^{1}(\Omega),
$$

which is a semigroup solution of the parabolic problem

$$
\begin{cases}\frac{\partial u}{\partial t}=\operatorname{div} \mathbf{a}(u, D u) & \text { in } Q_{T}=(0, T) \times \Omega \\ \frac{\partial u}{\partial \eta}=0 & \text { on } S_{T}=(0, T) \times \partial \Omega \\ u(0, x)=u_{0}(x) & \text { in } x \in \Omega .\end{cases}
$$

In a subsequent work [6] we shall define the notion of entropy solution for (1.2) and prove that the semigroup solution $u(t)$ is an entropy solution. Moreover, we shall also prove that entropy solutions of (1.2) are unique. As a technical tool both in this paper and in [6] we shall use the lower semi-continuity results proved in [20] for energy functionals whose density is a function $g(u, D u)$ convex in $D u$ and with a linear growth rate in $D u$.

Particular instances of these PDE's have been studied in [12], [13], [14] and [21], when $N=1$. Let us describe their results in some detail. In [12], [13], and [21] the authors considered the problem

$$
\begin{cases}\frac{\partial u}{\partial t}=\left(\varphi(u) \mathbf{b}\left(u_{x}\right)\right)_{x} & \text { in }(0, T) \times \mathbb{R} \\ u(0, x)=u_{0}(x) & \text { in } x \in \mathbb{R}\end{cases}
$$

corresponding to (1.2) when $N=1$ and $\mathbf{a}\left(u, u_{x}\right)=\varphi(u) \mathbf{b}\left(u_{x}\right)$, where $\varphi: \mathbb{R} \rightarrow$ $\mathbb{R}^{+}$is smooth and strictly positive, and $\mathbf{b}: \mathbb{R} \rightarrow \mathbb{R}$ is a smooth odd function such that $\mathbf{b}^{\prime}>0$ and $\lim _{s \rightarrow \infty} \mathbf{b}(s)=\mathbf{b}_{\infty}$. Such models appear as models for heat and mass transfer in turbulent fluids [8], or in the theory of phase transitions where the corresponding free energy functional has a linear growth rate with respect to the gradient [29]. As the authors observed, in general, there are no classical solutions of (1.2), they defined the notion of entropy solution and proved existence ([12]) and uniqueness ([21]) of entropy solutions of (1.3). Existence was proved for bounded strictly increasing initial conditions $u_{0}: \mathbb{R} \rightarrow$ $\mathbb{R}$ such that $\mathbf{b}\left(u_{0}^{\prime}\right) \in C(\mathbb{R})$ (where $\mathbf{b}\left(u_{0}^{\prime}\left(x_{0}\right)\right)=\mathbf{b}_{\infty}$ if $u_{0}$ is discontinuous at $x_{0}$ ), and $\mathbf{b}\left(u_{0}^{\prime}(x)\right) \rightarrow 0$ as $x \rightarrow \pm \infty$ [12]. The entropy condition was written in Oleinik's form and uniqueness was proved using Kruzhkov's method of doubling variables. of $\mathbb{R}$

In [14], [15], the author considered the Neumann problem in an interval

$$
\begin{cases}\frac{\partial u}{\partial t}=\left(\mathbf{a}\left(u, u_{x}\right)\right)_{x} & \text { in }(0, T) \times(0,1) \\ u_{x}(t, 0)=u_{x}(t, 1)=0 & \\ u(0, x)=u_{0}(x) & \text { in } x \in(0,1)\end{cases}
$$


for functions $\mathbf{a}(u, v)$ of class $C^{1, \alpha}([0, \infty) \times \mathbb{R})$ such that $\frac{\partial}{\partial v} \mathbf{a}(u, v)<0$ for any $(u, v) \in[0, \infty) \times \mathbb{R}, \mathbf{a}(u, 0)=0$ (and some other additional assumptions). After observing that there are no, in general, classical solutions of (1.2), the author associated an $m$-accretive operator to $-\left(\mathbf{a}\left(u, u_{x}\right)\right)_{x}$ with Neumann boundary conditions, and proved the existence and uniqueness of a semigroup solution of (1.4). However, the accretive operator generating the semigroup was not characterized in distributional terms. An example of the equations considered in [14], [15] is the so called plasma equation (see [23])

$$
\frac{\partial u}{\partial t}=\left(\frac{u^{5 / 2} u_{x}}{1+u\left|u_{x}\right|}\right)_{x} \quad \text { in } \quad(0, T) \times(0,1),
$$

where the initial condition $u_{0}$ is assumed to be positive. In this case $u$ represents the temperature of electrons and the form of the conductivity $\mathbf{a}\left(u, u_{x}\right)=\frac{u^{5 / 2} u_{x}}{1+u\left|u_{x}\right|}$ has the effect of limiting heat flux. However, existence and uniqueness results for higher dimensional problems were not considered. This will be the purpose of the present paper.

The case of equations of type

$$
u-\operatorname{div} \mathbf{a}(x, D u)=v \quad \text { in } \Omega,
$$

where $v \in L^{1}(\Omega)$, or the corresponding parabolic problem

$$
\frac{\partial u}{\partial t}=\operatorname{div} \mathbf{a}(x, D u) \quad \text { in } \quad(0, T) \times \Omega,
$$

where $\mathbf{a}(x, \xi)=\nabla_{\xi} f(x, \xi), f(x, \cdot)$ being a convex function of $\xi$ with linear growth as $\|\xi\| \rightarrow \infty$ has been considered in [2], [3] and [4] (see also [5]), where existence and uniqueness results of entropy solutions were proved.

The present work can be considered as an extension of the previous works to the case where a depends on $(u, D u)$ instead of $(x, D u)$. We treat in this paper the elliptic case, the parabolic problem being considered in [6]. Entropy or renormalized solutions for elliptic or parabolic problems of types (1.1), (1.2), or (1.7), when $f(u, \xi)$ or $f(x, \xi)$ has a growth of order $p>1$ as $\|\xi\| \rightarrow \infty$, were considered in [9], [16] and [17] (see also the references therein).

Finally, let us explain the plan of the paper. In Section 2 we recall some basic facts about functions of bounded variation, denoted by $B V(\Omega)$, Green's formula, and lower semi-continuity results for energy functionals defined in $B V(\Omega)$. In Section 3 we introduce the main assumptions on the underlying operator, and define the notion of entropy solutions for (1.1). In Section 4 we prove an existence and uniqueness result for the entropy solutions of (1.1) when the right hand side $v$ is in $L^{1}(\Omega)$. To prove existence we shall use the lower semi-continuity result for energy functionals whose density is a function $g(u, D u)$ convex in $D u$ and with a linear growth rate in $D u$ proved in [20], uniqueness will be proved by means of Kruzhkov's technique of doubling variables. Finally, in Section 5, we define an $m$-accretive operator associated 
to $-\operatorname{div} \mathbf{a}(u, D u)$ with Neumann boundary condition which, thus, generates a contraction semigroup in $L^{1}(\Omega)$, providing a solution of (1.2) in the semigroup sense. That semigroup solutions can be characterized in terms of entropy solutions will be the object of a subsequent paper [6].

Acknowledgements. The first and third authors have been supported by EC through the RTN Programme Nonlinear Partial Differential Equations Describing Front propagation and other Singular Phenomena, HPRN-CT-2002-00274, and by PNPGC project, reference BFM2002-01145. The second author acknowledges partial support by the Departament d'Universitats, Recerca i Societat de la Informació de la Generalitat de Catalunya and by PNPGC project, reference BFM2000-0962-C02-01.

\section{2. - Preliminaries}

We start with some notation. Here $\mathcal{L}^{N}$ and $\mathcal{H}^{N-1}$ are, respectively, the $N$ dimensional Lebesgue measure and the $(N-1)$-dimensional Hausdorff measure in $\mathbb{R}^{N}$.

Due to the linear growth condition on the Lagrangian, the natural energy space to study (1.1) is the space of functions of bounded variation. We recall briefly some facts about functions of bounded variation (for further information concerning functions of bounded variation we refer to [1], [24] or [30]).

A function $u \in L^{1}(\Omega)$ whose partial derivatives in the sense of distributions are measures with finite total variation in $\Omega$ is called a function of bounded variation. The class of such functions will be denoted by $B V(\Omega)$. Thus $u \in B V(\Omega)$ if and only if there are Radon measures $\mu_{1}, \ldots, \mu_{N}$ defined in $\Omega$ with finite total mass in $\Omega$ and

$$
\int_{\Omega} u D_{i} \varphi d x=-\int_{\Omega} \varphi d \mu_{i}
$$

for all $\varphi \in C_{0}^{\infty}(\Omega), i=1, \ldots, N$. Thus the gradient of $u$ is a vector valued measure with finite total variation

$$
|D u|(\Omega)=\sup \left\{\int_{\Omega} u \operatorname{div} \varphi d x: \varphi \in C_{0}^{\infty}\left(\Omega, \mathbb{R}^{N}\right),|\varphi(x)| \leq 1 \text { for } x \in \Omega\right\} .
$$

The space $B V(\Omega)$ is endowed with the norm

$$
\|u\|_{B V}=\|u\|_{L^{1}(\Omega)}+|D u|(\Omega) .
$$


For $u \in B V(\Omega)$, the gradient $D u$ is a Radon measure that decomposes into its absolutely continuous and singular parts $D u=D^{a} u+D^{s} u$. Then $D^{a} u=\nabla u \mathcal{L}^{N}$ where $\nabla u$ is the Radon-Nikodym derivative of the measure $D u$ with respect to the Lebesgue measure $\mathcal{L}^{N}$. Let us denote by $D^{s} u=\overrightarrow{D^{s} u}\left|D^{s} u\right|$ the polar decomposition of $D^{s} u$, where $\left|D^{s} u\right|$ is the total variation measure of $D^{s} u$. We also split $D^{s} u$ in two parts: the jump part $D^{j} u$ and the Cantor part $D^{c} u$. We denote by $S_{u}$ the set of all $x \in \Omega$ such that $x$ is not a Lebesgue point of $u$. We say that $x \in \Omega$ is an approximate jump point of $u$ if there exist $u^{+}(x) \neq u^{-}(x) \in \mathbb{R}$ and $v_{u}(x) \in S^{N-1}$ such that

$$
\begin{aligned}
& \lim _{\rho \downarrow 0} \frac{1}{\mathcal{L}^{N}\left(B_{\rho}^{+}\left(x, v_{u}(x)\right)\right)} \int_{B_{\rho}^{+}\left(x, v_{u}(x)\right)}\left|u(y)-u^{+}(x)\right| d y=0 \\
& \lim _{\rho \downarrow 0} \frac{1}{\mathcal{L}^{N}\left(B_{\rho}^{-}\left(x, v_{u}(x)\right)\right)} \int_{B_{\rho}^{-}\left(x, v_{u}(x)\right)}\left|u(y)-u^{-}(x)\right| d y=0,
\end{aligned}
$$

where

$$
B_{\rho}^{+}\left(x, v_{u}(x)\right)=\left\{y \in B_{\rho}(x):\left\langle y-x, v_{u}(x)\right\rangle>0\right\}
$$

and

$$
B_{\rho}^{-}\left(x, v_{u}(x)\right)=\left\{y \in B_{\rho}(x):\left\langle y-x, v_{u}(x)\right\rangle<0\right\} .
$$

We denote by $J_{u}$ the set of approximate jump points of $u$. $J_{u}$ is a Borel subset of $S_{u}$ and $\mathcal{H}^{N-1}\left(S_{u} \backslash J_{u}\right)=0$. We have

$$
D^{j} u=D^{s} u\left\llcorner J_{u} \quad \text { and } \quad D^{c} u=D^{s} u\left\llcorner\left(\Omega \backslash S_{u}\right) .\right.\right.
$$

It is well known (see for instance [1]) that

$$
D^{j} u=\left(u^{+}-u^{-}\right) v_{u} \mathcal{H}^{N-1}\left\llcorner J_{u} .\right.
$$

Moreover, if $x \in J_{u}$, then $v_{u}(x)=\frac{D u}{|D u|}(x), \frac{D u}{|D u|}$ being the Radon-Nikodym derivative of $D u$ with respect to its total variation $|D u|$.

Let $\Omega$ be a bounded open subset of $\mathbb{R}^{N}$. Given a Borel function $g$ : $\Omega \times \mathbb{R} \times \mathbb{R}^{N} \rightarrow \mathbb{R}^{+}$such that

$$
C\|\xi\|-D \leq g(x, z, \xi) \leq M(1+\|\xi\|) \quad \forall(x, z, \xi) \in \Omega \times \mathbb{R} \times \mathbb{R}^{N}
$$

for some constants $C>0, M \geq 0$, we consider the energy functional

$$
G(u):=\int_{\Omega} g(x, u(x), \nabla u(x)) d x
$$

defined in the Sobolev space $W^{1,1}(\Omega)$. In order to get an integral representation of the relaxed energy associated with $G$, i.e.,

$$
\mathcal{G}(u):=\inf _{\left\{u_{n}\right\}}\left\{\liminf _{n \rightarrow \infty} G\left(u_{n}\right): u_{n} \in W^{1,1}(\Omega), u_{n} \rightarrow u \in L^{1}(\Omega)\right\}
$$


Dal Maso in [20] introduced the following functional for $u \in B V(\Omega)$ :

$$
\begin{aligned}
\mathcal{R}_{g}(u):= & \int_{\Omega} g(x, u(x), \nabla u(x)) d x+\int_{\Omega} g^{0}\left(x, u(x), \frac{D u}{|D u|}(x)\right)\left|D^{c} u\right| \\
& +\int_{J_{u}}\left(\int_{u_{-}(x)}^{u_{+}(x)} g^{0}\left(x, s, v_{u}(x)\right) d s\right) d \mathcal{H}^{N-1}(x),
\end{aligned}
$$

where the recession function $g^{0}$ of $g$ is defined as

$$
g^{0}(x, z, \xi)=\lim _{t \rightarrow 0^{+}} \operatorname{tg}\left(x, z, \frac{\xi}{t}\right)
$$

It is clear that the function $g^{0}(x, z, \xi)$ is positively homogeneous of degree one in $\xi$, i.e.

$$
g^{0}(x, z, s \xi)=s g^{0}(x, z, \xi) \quad \text { for all } z, \xi \text { and } s>0
$$

Let us describe a different way of writing the functional $\mathcal{R}_{g}(u)$. Let us consider the function $\left.\left.\tilde{g}: \Omega \times \mathbb{R} \times \mathbb{R}^{N} \times\right]-\infty, 0\right] \rightarrow \mathbb{R}$ defined as

$$
\tilde{g}(x, z, \xi, t):= \begin{cases}-g\left(x, z,-\frac{\xi}{t}\right) t & \text { if } t<0 \\ g^{0}(x, z, \xi) & \text { if } t=0 .\end{cases}
$$

As it is proved in [20], if $g$ is a Borel function satisfying (2.3) and $g(x, z, \cdot)$ is convex in $\mathbb{R}^{N}$ for all $(x, z) \in \Omega \times \mathbb{R}$, then one has

$$
\begin{aligned}
\mathcal{R}_{g}(u) & =\int_{\Omega \times \mathbb{R}} \tilde{g}\left((x, s), \frac{d \alpha_{u}}{d\left|\alpha_{u}\right|}(x, s)\right) d\left|\alpha_{u}\right|(x, s) \\
& =\int_{\Omega \times \mathbb{R}} \tilde{g}((x, s), v[(x, s) ; N(u)]) d \mathcal{H}^{N}(x, s),
\end{aligned}
$$

where $\alpha_{u}=D \chi_{N(u)}$, with $N(u):=\left\{(x, s) \in \mathbb{R} \times \Omega: s<u_{+}(x)\right\}$ and $v[(x, s) ; N(u)]$ is the interior normal to $N(u)$ at $(s, x)$ if it exists, and $\nu[(x, s) ; N(u)]=0$ otherwise.

In [20] Dal Maso proved the following result:

THEOREM 2.1. Let $\Omega$ be a bounded open subset of $\mathbb{R}^{N}$. Let $g: \Omega \times \mathbb{R} \times \mathbb{R}^{N} \rightarrow \mathbb{R}$ be a continuous function satisfying $(2.3), g^{0}$ exists and such that $g(x, z, \cdot)$ is convex in $\mathbb{R}^{N}$. Then, $\mathcal{G}(u)=\mathcal{R}_{g}(u)$ for all $u \in B V(\Omega)$ and $\mathcal{R}_{g}(u)$ is lower semi-continuous respect to the $L^{1}(\Omega)$-convergence.

We need to consider the following truncature functions. For $a<b$, let $T_{a, b}(r):=\max (\min (b, r), a)$. It is usual to denote $T_{k}=T_{-k, k}$. 
Proposition 2.2. Let $\Omega$ be a bounded open subset of $\mathbb{R}^{N}$. Let $g: \Omega \times \mathbb{R} \times \mathbb{R}^{N} \rightarrow$ $\mathbb{R}$ be a continuous function satisfying $(2.3), g^{0}$ exists and such that $g(x, z, \cdot)$ is convex in $\mathbb{R}^{N}$. Let us define the functional

$$
R_{g}^{a, b}(u)=\int_{\Omega} g\left(x, u(x), \nabla T_{a, b} u(x)\right) d x, \quad u \in W^{1,1}(\Omega) .
$$

For $u \in B V(\Omega)$, let

$$
\begin{aligned}
\mathcal{R}_{g}^{a, b}(u):= & \int_{\Omega} \int_{a}^{b} \tilde{g}((x, s), \nu[(x, s) ; N(u)]) d \mathcal{H}^{N-1}(x) d s \\
& +\int_{[u \leq a]}(g(x, u(x), 0)-g(x, a, 0)) d x \\
& +\int_{[u \geq b]}(g(x, u(x), 0)-g(x, b, 0)) d x .
\end{aligned}
$$

Then $\mathcal{R}_{g}^{a, b}(u)$ is lower semi-continuous with respect to the $L^{1}(\Omega)$-convergence, and $\mathcal{R}_{g}^{a, b}$ coincides with the lower semi-continuous envelope of $R_{g}^{a, b}$.

Proof. Observe that we have

$$
\mathcal{R}_{g}\left(T_{a, b}(u)\right)=\int_{\Omega} \int_{a}^{b} \tilde{g}((x, s), v[(x, s) ; N(u)]) d \mathcal{H}^{N-1}(x) d s .
$$

Hence, we may write

$$
\begin{aligned}
\mathcal{R}_{g}^{a, b}(u)= & \mathcal{R}_{g}\left(T_{a, b}(u)\right)+\int_{[u \leq a]}(g(x, u(x), 0)-g(x, a, 0)) d x \\
& +\int_{[u \geq b]}(g(x, u(x), 0)-g(x, b, 0)) d x .
\end{aligned}
$$

Since the functional $\mathcal{R}_{g}$ is lower semi-continuous with respect to $L^{1}(\Omega)$ convergence (Theorem 2.1), we conclude that $\mathcal{R}_{g}^{a, b}$ is also lower semi-continuous. Moreover, if $u \in W^{1,1}(\Omega)$, we have

$$
\begin{aligned}
R_{g}^{a, b}(u)= & \int_{[a<u<b]} g(x, u(x), \nabla u(x)) d x+\int_{[u \leq a]} g(x, u(x), 0) d x+\int_{[u \geq b]} g(x, u(x), 0) d x \\
= & \int_{[a<u<b]} g(x, u(x), \nabla u(x)) d x+\int_{[u \leq a]} g(x, a, 0) d x \\
& +\int_{[u \geq b]} g(x, b, 0) d x+\int_{[u \leq a]}(g(x, u(x), 0)-g(x, a, 0)) d x \\
& +\int_{[u \geq b]}(g(x, u(x), 0)-g(x, b, 0)) d x \\
= & \mathcal{R}_{g}\left(T_{a, b}(u)\right)+\int_{[u \leq a]}(g(x, u(x), 0)-g(x, a, 0)) d x \\
& +\int_{[u \geq b]}(g(x, u(x), 0)-g(x, b, 0)) d x=\mathcal{R}_{g}^{a, b}(u) .
\end{aligned}
$$

Therefore, $\mathcal{R}_{g}^{a, b}$ is a lower semi-continuous extension of $R_{g}^{a, b}$ to $B V(\Omega)$. 
Let $g: \mathbb{R} \times \mathbb{R}^{N} \rightarrow \mathbb{R}$ be a continuous function satisfying

$$
C\|\xi\|-D \leq g(z, \xi) \leq M(1+\|\xi\|) \quad \forall(z, \xi) \in \times \mathbb{R} \times \mathbb{R}^{N},
$$

for some constants $C>0, M \geq 0$. Given $u \in B V(\Omega)$, let us define the measures

$$
\mathcal{R}_{g}(u, \phi):=\int_{\mathbb{R}} \int_{\Omega} \phi(x) \tilde{g}(s, \nu[(s, x) ; N(u)]) d \mathcal{H}^{N-1}(x) d s
$$

and

$$
\begin{aligned}
\mathcal{R}_{g}^{a, b}(u, \phi):= & \int_{a}^{b} \int_{\Omega} \phi(x) \tilde{g}(s, \nu[(s, x) ; N(u)]) d \mathcal{H}^{N-1}(x) d s \\
& +\int_{[u \leq a]} \phi(x)(g(u(x), 0)-g(a, 0)) d x \\
& +\int_{[u \geq b]} \phi(x)(g(u(x), 0)-g(b, 0)) d x
\end{aligned}
$$

for any $\phi \in C(\bar{\Omega})$. For simplicity, we shall write

$$
\mathcal{R}_{g}(u, \phi)=\int_{\Omega} \phi(x) g(u, D u)
$$

and

$$
\mathcal{R}_{g}^{a, b}(u, \phi)=\int_{\Omega} \phi(x) g\left(u, D T_{a, b}(u)\right) .
$$

The singular parts with respect to the Lebesgue measure $\mathcal{L}^{N}$ of these measures will be denoted by

$$
\left(\mathcal{R}_{g}\right)^{s}(u, \phi)=\int_{\Omega} \phi(x) g(u, D u)^{s}
$$

and

$$
\left(\mathcal{R}_{g}^{a, b}\right)^{s}(u, \phi)=\int_{\Omega} \phi(x) g\left(u, D T_{a, b}(u)\right)^{s},
$$

respectively.

We shall need several results from [7] (see also [26]) in order to give a sense to the integrals of bounded vector fields with divergence in $L^{p}$ integrated with respect to the gradient of a $B V$ function. Let $p \geq 1$ and $p^{\prime} \geq 1$ be such that $\frac{1}{p}+\frac{1}{p^{\prime}}=1$. Following [7], let

$$
X_{p}(\Omega)=\left\{\mathbf{z} \in L^{\infty}\left(\Omega, \mathbb{R}^{N}\right): \operatorname{div}(\mathbf{z}) \in L^{p}(\Omega)\right\} .
$$

If $\mathbf{z} \in X_{p}(\Omega)$ and $w \in B V(\Omega) \cap L^{p^{\prime}}(\Omega)$ we define the functional (z, $\left.D w\right)$ : $C_{0}^{\infty}(\Omega) \rightarrow \mathbb{R}$ by the formula

$$
\langle(\mathbf{z}, D w), \varphi\rangle:=-\int_{\Omega} w \varphi \operatorname{div}(\mathbf{z}) d x-\int_{\Omega} w \mathbf{z} \cdot \nabla \varphi d x .
$$


Then $(\mathbf{z}, D w)$ is a Radon measure in $\Omega$,

$$
\int_{\Omega}(\mathbf{z}, D w)=\int_{\Omega} \mathbf{z} \cdot \nabla w d x \quad \forall w \in W^{1,1}(\Omega) \cap L^{\infty}(\Omega)
$$

and

$$
\left|\int_{B}(\mathbf{z}, D w)\right| \leq \int_{B}|(\mathbf{z}, D w)| \leq\|\mathbf{z}\|_{\infty} \int_{B}|D w|
$$

for any Borel set $B \subseteq \Omega$. Moreover, $(\mathbf{z}, D w)$ is absolutely continuous with respect to $|D w|$ with Radon-Nikodym derivative $\theta(\mathbf{z}, D w, x)$ which is a $|D w|$ measurable function from $\Omega$ to $\mathbb{R}$ such that

$$
\int_{B}(\mathbf{z}, D w)=\int_{B} \theta(\mathbf{z}, D w, x)|D w|
$$

for any Borel set $B \subseteq \Omega$. We also have that

$$
\|\theta(\mathbf{z}, D w, .)\|_{L^{\infty}(\Omega,|D w|)} \leq\|\mathbf{z}\|_{L^{\infty}\left(\Omega, \mathbb{R}^{N}\right)} .
$$

By writing

$$
\mathbf{z} \cdot D^{s} u:=(\mathbf{z}, D u)-(\mathbf{z} \cdot \nabla u) d \mathcal{L}^{N},
$$

we see that $\mathbf{z} \cdot D^{s} u$ is a bounded measure. Furthermore, in [26] it is proved that $\mathbf{z} \cdot D^{s} u$ is absolutely continuous with respect to $\left|D^{s} u\right|$ (thus, it is a singular measure with respect to $\mathcal{L}^{N}$ ), and

$$
\left|\mathbf{z} \cdot D^{s} u\right| \leq\|\mathbf{z}\|_{\infty}\left|D^{s} u\right| .
$$

As a consequence of Theorem 2.4 of [7], we have:

$$
\text { If } \quad \mathbf{z} \in X_{p}(\Omega) \cap C\left(\Omega, \mathbb{R}^{N}\right), \quad \text { then } \quad \mathbf{z} \cdot D^{s} u=\left(\mathbf{z} \cdot \overrightarrow{D^{s} u}\right) d\left|D^{s} u\right| .
$$

In [7], a weak trace on $\partial \Omega$ of the normal component of $\mathbf{z} \in X_{p}(\Omega)$ is defined. Concretely, it is proved that there exists a linear operator $\gamma: X_{p}(\Omega) \rightarrow$ $L^{\infty}(\partial \Omega)$ such that

$$
\begin{gathered}
\|\gamma(\mathbf{z})\|_{\infty} \leq\|\mathbf{z}\|_{\infty} \\
\gamma(\mathbf{z})(x)=\mathbf{z}(x) \cdot v(x) \quad \text { for all } x \in \partial \Omega \text { if } \quad \mathbf{z} \in C^{1}\left(\bar{\Omega}, \mathbb{R}^{N}\right) .
\end{gathered}
$$

We shall denote $\gamma(\mathbf{z})(x)$ by $[\mathbf{z}, v](x)$. Moreover, the following Green's formula, relating the function $[\mathbf{z}, v]$ and the measure $(\mathbf{z}, D w)$, for $\mathbf{z} \in X_{p}(\Omega)$ and $w \in B V(\Omega) \cap L^{p^{\prime}}(\Omega)$, is established:

$$
\int_{\Omega} w \operatorname{div}(\mathbf{z}) d x+\int_{\Omega}(\mathbf{z}, D w)=\int_{\partial \Omega}[\mathbf{z}, \nu] w d \mathcal{H}^{N-1} .
$$




\section{3. - Basic assumptions. The notion of entropy solution for the elliptic problem}

This section deals with the elliptic problem

$$
\begin{cases}v=-\operatorname{div} \mathbf{a}(u, D u) & \text { in } \Omega \\ \frac{\partial u}{\partial \eta}=0 & \text { on } \partial \Omega .\end{cases}
$$

Here we assume that $\Omega$ is an open bounded set in $\mathbb{R}^{N}$, with boundary $\partial \Omega$ of class $C^{1}$, and the Lagrangian $f: \mathbb{R} \times \mathbb{R}^{N} \rightarrow \mathbb{R}$ satisfies the following assumptions, which we shall refer collectively as $(\mathrm{H})$ :

$\left(\mathrm{H}_{1}\right) \quad f$ is continuous on $\mathbb{R} \times \mathbb{R}^{N}$ and is a convex differentiable function of $\xi$ such that $\nabla_{\xi} f(z, \xi) \in C\left(\mathbb{R} \times \mathbb{R}^{N}\right)$. Further we require $f$ to satisfy the linear growth condition

$$
C_{0}\|\xi\|-D_{0} \leq f(z, \xi) \leq M(\|\xi\|+1) .
$$

for any $(z, \xi) \in \mathbb{R} \times \mathbb{R}^{N},|z| \leq R$ and some positive constants $C_{0}, D_{0}, M$ depending on $R$. Moreover, we assume that $f^{0}$ exists.

We consider the function $\mathbf{a}(z, \xi)=\nabla_{\xi} f(z, \xi)$ associated to the Lagrangian $f$. By the convexity of $f$, we have

$$
\mathbf{a}(z, \xi) \cdot(\eta-\xi) \leq f(z, \eta)-f(z, \xi), \quad \forall z \in \mathbb{R}, \quad \forall \xi, \eta \in \mathbb{R}^{N},
$$

and the following monotonicity condition is satisfied

$$
(\mathbf{a}(z, \eta)-\mathbf{a}(z, \xi)) \cdot(\eta-\xi) \geq 0, \quad \forall z \in \mathbb{R}, \quad \forall \xi, \eta \in \mathbb{R}^{N} .
$$

Moreover, it is easy to see that

$$
|\mathbf{a}(z, \xi)| \leq M \quad \forall(z, \xi) \in \mathbb{R} \times \mathbb{R}^{N},|z| \leq R .
$$

We also assume that $\mathbf{a}(z, 0)=0$ for all $z \in \mathbb{R}$. We consider the function $h: \mathbb{R} \times \mathbb{R}^{N} \rightarrow \mathbb{R}$ defined by

$$
h(z, \xi):=\mathbf{a}(z, \xi) \cdot \xi
$$

By (3.4), we have

$$
h(z, \xi) \geq 0 \quad \forall \xi \in \mathbb{R}^{N}, \quad z \in \mathbb{R} .
$$

Moreover, from (3.3) and (3.2), it follows that

$$
C_{0}\|\xi\|-D_{1} \leq h(z, \xi) \leq M\|\xi\|
$$

for any $(z, \xi) \in \mathbb{R} \times \mathbb{R}^{N},|z| \leq R$, where $D_{1}$ is a positive constant depending on $R, C_{0}$ and $M$ being as above. 
$\left(\mathrm{H}_{2}\right)$ We assume that $\frac{\partial \mathbf{a}}{\partial \xi_{i}}(z, \xi) \in C\left(\mathbb{R} \times \mathbb{R}^{N}\right)$ for any $i=1, \ldots, N$.

This assumption is not necessary for the case of separated variables described in Remark 3.1.

We assume that

$\left(\mathrm{H}_{3}\right) \quad h(z, \xi)=h(z,-\xi)$, for all $z \in \mathbb{R}$ and $\xi \in \mathbb{R}^{N}$ and $h^{0}$ exists.

Observe that we have

$$
C_{0}\|\xi\| \leq h^{0}(z, \xi) \leq M\|\xi\| \quad \text { for any }(z, \xi) \in \mathbb{R} \times \mathbb{R}^{N},|z| \leq R .
$$

$\left(\mathrm{H}_{4}\right) f^{0}(z, \xi)=h^{0}(z, \xi)$, for all $\xi \in \mathbb{R}^{N}$ and all $z \in \mathbb{R}$.

$\left(\mathrm{H}_{5}\right) \mathbf{a}(z, \xi) \cdot \eta \leq h^{0}(z, \eta)$ for all $\xi, \eta \in \mathbb{R}^{N}$, and all $z \in \mathbb{R}$.

$\left(\mathrm{H}_{6}\right)$ We assume that $h^{0}(z, \xi)$ can be written in the form $h^{0}(z, \xi)=\varphi(z) \psi^{0}(\xi)$ with $\varphi$ a $C^{1}$-function such that for any $R>0$, we have $\varphi(z)>\alpha_{R}>0$ for all $z \in \mathbb{R},|z| \leq R$, and $\psi^{0}$ being a convex function homogeneous of degree 1 .

$\left(\mathrm{H}_{7}\right)$ For any $R>0$, there is a constant $C>0$ such that

$$
|(\mathbf{a}(z, \xi)-\mathbf{a}(\hat{z}, \xi)) \cdot(\xi-\hat{\xi})| \leq C|z-\hat{z}|\|\xi-\hat{\xi}\|
$$

for any $(z, \xi),(\hat{z}, \hat{\xi}) \in \mathbb{R} \times \mathbb{R}^{N},|z|,|\hat{z}| \leq R$.

Observe that, by the monotonicity condition (3.4) and using (3.8), it follows that

$$
(\mathbf{a}(z, \xi)-\mathbf{a}(\hat{z}, \hat{\xi})) \cdot(\xi-\hat{\xi}) \geq-C|z-\hat{z}|\|\xi-\hat{\xi}\|
$$

for any $(z, \xi),(\hat{z}, \hat{\xi}) \in \mathbb{R} \times \mathbb{R}^{N},|z|,|\hat{z}| \leq R$.

Let us observe that under assumptions $\left(\mathrm{H}_{4}\right)$ and $\left(\mathrm{H}_{6}\right)$, applying the chain rule for BV-functions (see [1]), we have

$$
\mathcal{R}_{f}(u)=\int_{\Omega} f(u, \nabla u) d x+\psi^{0}\left(\frac{D u}{|D u|}\right)\left|D^{s} J_{\varphi}(u)\right|,
$$

where $J_{\varphi}(r)=\int_{0}^{r} \varphi(s) d s$.

REMARK 3.1. An important particular case of Lagrangian $f$ satisfying all assumptions $(\mathrm{H})$ but $\left(\mathrm{H}_{2}\right)$, is the one given by $f(z, \xi)=\varphi(z) \psi(\xi)$ with $\varphi$ a $C^{1}$-function such that for any $R>0$, we have $\varphi(z)>\alpha_{R}>0$ for all $z \in \mathbb{R}$, $|z| \leq R$, and $\psi$ a convex $C^{1}$-function such that

$$
C_{0}\|\xi\|-D_{0} \leq \psi(\xi) \leq M(\|\xi\|+1) \quad \forall \xi \in \mathbb{R}^{N},
$$

and there exists

$$
\psi^{0}(\xi)=\lim _{t \rightarrow 0^{+}} t \psi\left(\frac{\xi}{t}\right)
$$


In this case, if $\mathbf{b}(\xi):=\nabla \psi(\xi)$, we have $\mathbf{a}(z, \xi)=\varphi(z) \mathbf{b}(\xi)$, and $h(z, \xi)=$ $\mathbf{a}(z, \xi) \cdot \xi=\varphi(z) \mathbf{b}(\xi) \cdot \xi$. Then, in order to have that $(\mathrm{H})$ holds we need to assume that:

(i) $\mathbf{b}(-\xi) \cdot(-\xi)=\mathbf{b}(\xi) \cdot \xi \geq 0$ for all $\xi \in \mathbb{R}^{N}$ and there exists

$$
\lim _{t \rightarrow 0^{+}} \mathbf{b}\left(\frac{\xi}{t}\right) \cdot \xi=\psi^{0}(\xi)
$$

(ii) $\mathbf{b}(\xi) \cdot \eta \leq \psi^{0}(\xi) \cdot \eta$ for all $\xi, \eta \in \mathbb{R}^{N}$.

We note that in this case $\left(\mathrm{H}_{2}\right)$ is not necessary to obtain existence and uniqueness of solutions for problem (1.2). Let us prove that $\left(\mathrm{H}_{7}\right)$ holds. Indeed, by applying the mean value Theorem, we have

$$
\begin{aligned}
& |(\mathbf{a}(z, \xi)-\mathbf{a}(\hat{z}, \xi)) \cdot(\xi-\hat{\xi})|=|(\varphi(z)-\varphi(\hat{z}))||\mathbf{b}(\xi) \cdot(\xi-\hat{\xi})| \\
\leq & \sup _{\tau \in[0,1]}\left|\varphi^{\prime}(\tau z+(1-\tau) \hat{z})\right| M|z-\hat{z}|\|\xi-\hat{\xi}\| \leq C|z-\hat{z}|\|\xi-\hat{\xi}\|,
\end{aligned}
$$

for some constant $C>0$ depending on $R$ and any $(z, \xi),(\hat{z}, \xi) \in \mathbb{R} \times \mathbb{R}^{N}$, $|z|,|\hat{z}| \leq R$.

REMARK 3.2. There are physical models for plasma fusion by inertial confinement in which the temperature evolution of the electrons satisfies an equation of type (1.2), where $\mathbf{a}(z, \xi)=\frac{|z|^{5 / 2} \xi}{1+|z| \xi \mid}$ which corresponds to $f(z, \xi)=$ $|z|^{3 / 2}|\xi|-|z|^{1 / 2} \ln (1+|z||\xi|)$ [23], (see also [14] for a mathematical study in the one-dimensional case). It is easy to check that $\left(\mathrm{H}_{1}\right)$ (in particular (3.2) and (3.7)) holds for any $(z, \xi) \in \mathbb{R} \times \mathbb{R}^{N}$ with $z \in[a, R], a>0$, the constants in (3.2) and (3.7) depending on $a, R$. Note that $\left(\mathrm{H}_{2}\right)$ also holds. We also observe that $h^{0}(z, \xi)=|z|^{3 / 2}|\xi|$ and $\left(\mathrm{H}_{3}\right)-\left(\mathrm{H}_{6}\right)$ hold. Finally, to check $\left(\mathrm{H}_{7}\right)$ we observe that

$$
\frac{\partial \mathbf{a}}{\partial z}(z, \xi)=\frac{5}{2} \frac{z^{3 / 2} \xi}{1+z|\xi|}-\frac{z^{5 / 2}|\xi| \xi}{(1+z|\xi|)^{2}}
$$

and therefore

$$
\left|\frac{\partial \mathbf{a}}{\partial z}(z, \xi)\right| \leq \frac{7}{2} z^{1 / 2}
$$

for any $z \in[a, R]$ and any $\xi \in \mathbb{R}^{N}$. It follows that

$$
|\mathbf{a}(z, \xi)-\mathbf{a}(\hat{z}, \xi)| \leq \frac{7}{2} R^{1 / 2}|z-\hat{z}|
$$

for any $z \in[a, R]$ and any $\xi \in \mathbb{R}^{N}$. Thus $\left(\mathrm{H}_{7}\right)$ also holds for the values of $z \in[a, R]$ and $\xi \in \mathbb{R}^{N}$. In this case, the results below will prove existence and uniqueness of entropy solutions of (1.1) for any initial condition $v \in L^{\infty}(\Omega)$ such that $v(x) \geq a>0$ for some $a>0$. 
We need to consider the function space

$$
T B V(\Omega):=\left\{u \in L^{1}(\Omega): T_{k}(u) \in B V(\Omega), \quad \forall k>0\right\},
$$

and to give a sense to the Radon-Nikodym derivative $\nabla u$ of a function $u \in$ $T B V(\Omega)$. Notice that the function space $T B V(\Omega)$ is closely related to the space $G B V(\Omega)$ of generalized functions of bounded variation introduced by $E$. Di Giorgi and L. Ambrosio ([22], see also [1]). In [3] we give the following results.

Lemma 3.3. For every $u \in T B V(\Omega)$ there exists a unique measurable function $v: \Omega \rightarrow \mathbb{R}^{N}$ such that

$$
\nabla T_{k}(u)=v \chi_{\{|u|<k\}} \quad \mathcal{L}^{N}-\text { a.e. }
$$

LEMmA 3.4. If $u \in T B V(\Omega)$, then $p(u) \in B V(\Omega)$ for every Lipschitz continuous function $p: \mathbb{R} \rightarrow \mathbb{R}$ satisfying $p^{\prime}(s)=0$ for $|s|$ large enough. Moreover, $\nabla p(u)=p^{\prime}(u) \nabla u \quad \mathcal{L}^{N}$-a.e.

Thanks to Lemma 3.3 we define $\nabla u$ for a function $u \in T B V(\Omega)$ as the unique function $v$ which satisfies (3.11). This notation will be used throughout in the sequel.

We introduce the following concept of solution for problem (3.1)

Definition 3.5. Given $v \in L^{1}(\Omega)$, we say that $u \in L^{1}(\Omega)$ is an entropy solution of (3.1) if $u \in T B V(\Omega), \mathbf{a}(u, \nabla u) \in X_{1}(\Omega)$ and satisfies:

$$
\begin{gathered}
v=-\operatorname{div} \mathbf{a}(u, \nabla u) \quad \text { in } \mathcal{D}^{\prime}(\Omega), \\
\left(\mathbf{a}(u, \nabla u), D T_{a, b}(u)\right) \geq h\left(u, D T_{a, b}(u)\right) \quad \text { as measures } \forall a<b, \\
{[\mathbf{a}(u, \nabla u), v]=0 \quad \mathcal{H}^{N-1}-\text { a.e. on } \partial \Omega .}
\end{gathered}
$$

Recall that, since $h(z, 0)=0$ for any $z \in \mathbb{R}, h\left(u, D T_{a, b}(u)\right)$ is the density of the measure $\mathcal{R}_{h}^{a, b}(u)=\mathcal{R}_{h}\left(T_{a, b}(u)\right)$. Our assumptions on $h$ permit to apply the results described by Theorem 2.1, and Proposition 2.2. Hence, it has sense that we write $h\left(u, D T_{a, b}(u)\right)$ in (3.13) since it coincides with $h\left(T_{a, b}(u), D T_{a, b}(u)\right)$.

Observe that (3.13) is equivalent to

$$
\mathbf{a}(u, \nabla u) \cdot D^{s} T_{a, b}(u) \geq\left(\mathcal{R}_{f}^{a, b}\right)^{s}(u) \quad \text { as measures } \forall a<b .
$$

Let us also observe that we require that $\mathbf{a}(u, \nabla u) \in X_{1}(\Omega)$, and, thus, $\mathbf{a}(u, \nabla u) \in L^{\infty}\left(\Omega, \mathbb{R}^{N}\right)$. This is reasonable only if we are able to prove that solutions of (1.1) satisfy it, and, indeed, we shall prove it in Theorem 4.1 under different sets of assumptions.

We have the following characterization of entropy solutions. 
Proposition 3.6. Let $v \in L^{1}(\Omega)$ and let $u \in T B V(\Omega)$ with $\mathbf{a}(u, \nabla u) \in X_{1}(\Omega)$, satisfying (3.12) and (3.14). Then $u$ is an entropy solution of (3.1) (i.e., satisfies (3.13)) if and only if $u$ satisfies

$$
\int_{\Omega} \phi h\left(u, D T_{a, b}(u)\right)+\int_{\Omega} T_{a, b}(u) \mathbf{a}(u, \nabla u) \cdot \nabla \phi d x \leq \int_{\Omega} \phi v T_{a, b}(u) d x
$$

for any $\phi \in \mathcal{D}(\Omega), \phi \geq 0$.

Proof. Assume that $u$ is an entropy solution of (3.1). Multiplying (3.12) by $T_{a, b}(u) \phi$, integrating by parts and using (3.13) and (3.14), we obtain (3.16). Similarly, from (3.16), (3.13) and (3.14), we have

$$
\begin{aligned}
\int_{\Omega} \phi h\left(u, D T_{a, b}(u)\right) & +\int_{\Omega} T_{a, b}(u) \mathbf{a}(u, \nabla u) \cdot \nabla \phi d x \\
& \leq \int_{\Omega} \phi v T_{a, b}(u) d x \\
& =-\int_{\Omega} \operatorname{div} \mathbf{a}(u, \nabla u) T_{a, b}(u) \phi d x \\
& =\int_{\Omega}\left(\mathbf{a}(u, \nabla u), D\left(T_{a, b}(u) \phi\right)\right) \\
& =\int_{\Omega} \phi\left(\mathbf{a}(u, \nabla u), D T_{a, b}(u)\right)+\int_{\Omega} T_{a, b}(u) \mathbf{a}(u, \nabla u) \cdot \nabla \phi d x
\end{aligned}
$$

Hence,

$$
\int_{\Omega} \phi h\left(u, D T_{a, b}(u)\right) \leq \int_{\Omega} \phi\left(\mathbf{a}(u, \nabla u), D T_{a, b}(u)\right)
$$

for all $\phi \in \mathcal{D}(\Omega), \phi \geq 0$. This implies (3.13).

\section{4. - Existence and uniqueness of entropy solutions} result.

This section is devoted to prove the following existence and uniqueness

THEOREM 4.1. Assume that assumptions $(H)$ hold. Then, for any $v \in L^{\infty}(\Omega)$ there exists a unique entropy solution $u \in T B V(\Omega) \cap L^{\infty}(\Omega)$ of the problem

$$
\begin{cases}u-\operatorname{div} \mathbf{a}(u, D u)=v & \text { in } \Omega \\ \frac{\partial u}{\partial \eta}=0 & \text { on } \partial \Omega .\end{cases}
$$

Moreover, if we assume that the bound (3.5) holds for any $(z, \xi) \in \mathbb{R} \times \mathbb{R}^{N}$, then (4.1) has a unique entropy solution $u \in T B V(\Omega)$ for any $v \in L^{1}(\Omega)$. 
Proof. Step 1. Existence of entropy solutions. Let $v \in L^{1}(\Omega)$, and let $v_{n} \in L^{\infty}(\Omega)$ be such that $v_{n} \rightarrow v$ in $L^{1}(\Omega)$. If $v \in L^{\infty}(\Omega)$ we take $v_{n}=v$. Let $\beta(r)=\arctan (r)$. For every $n \in \mathbb{N}$, consider

$$
\mathbf{a}_{n}(z, \xi):=\mathbf{a}(z, \xi)+\frac{1}{n} \beta^{\prime}(z) \xi,
$$

and, then for each $m \in \mathbb{N}$,

$$
\mathbf{a}_{n, m}(z, \xi):=\mathbf{a}_{n}(z, \xi)+\frac{1}{m} \xi
$$

Let us fix $n \in \mathbb{N}$. Since $\mathbf{a}_{n, m}$ satisfies the classical Leray-Lions conditions ([28]), we know that for any $m \in \mathbb{N}$ there exists $u_{n, m} \in W^{1,2}(\Omega)$ such that

$$
\int_{\Omega} w\left(u_{n, m}-v_{n}\right) d x=-\int_{\Omega} \mathbf{a}_{n, m}\left(u_{n, m}, \nabla u_{n, m}\right) \cdot \nabla w d x \quad \forall w \in W^{1,2}(\Omega),
$$

that is

$$
u_{n, m}-\operatorname{div} \mathbf{a}_{n, m}\left(u_{n, m}, \nabla u_{n, m}\right)=v_{n} \quad \text { in } \mathcal{D}^{\prime}(\Omega)
$$

$$
\left[\mathbf{a}_{n, m}\left(u_{n, m}, \nabla u_{n, m}\right), v\right]=0 \quad \text { in } \partial \Omega
$$

Let us prove that

$$
\left\|u_{n, m}\right\|_{\infty} \leq\left\|v_{n}\right\|_{\infty} \quad \text { for all } m \in \mathbb{N} .
$$

For that, take $w=\left(u_{n, m}-\left\|v_{n}\right\|_{\infty}\right)^{+}$as test function in (4.2), we obtain

$$
\int_{\Omega}\left(u_{n, m}-\left\|v_{n}\right\|_{\infty}\right)^{+}\left(u_{n, m}-v_{n}\right) d x \leq 0 .
$$

Hence,

$\int_{\left\{u_{n, m}>\left\|v_{n}\right\|_{\infty}\right\}}\left(u_{n, m}-\left\|v_{n}\right\|_{\infty}\right)^{2} d x \leq \int_{\left\{u_{n, m}>\left\|v_{n}\right\|_{\infty}\right\}}\left(u_{n, m}-\left\|v_{n}\right\|_{\infty}\right)\left(u_{n, m}-v_{n}\right) d x \leq 0$.

Consequently, $u_{n, m} \leq\left\|v_{n}\right\|_{\infty}$ a.e. in $\Omega$. Similarly, taking $w=\left(u_{n, m}+\right.$ $\left.\left\|v_{n}\right\|_{\infty}\right)^{-}:=\min \left(u_{n, m}+\left\|v_{n}\right\|_{\infty}, 0\right)$ as test function, we get $-\left\|v_{n}\right\|_{\infty} \leq u_{n, m}$ a.e. in $\Omega$. Both inequalities prove (4.4). Now, multiplying (4.3) by $\beta\left(u_{n, m}\right)$ we obtain

$$
\frac{1}{n} \int_{\Omega}\left|\nabla \beta\left(u_{n, m}\right)\right|^{2} \leq \frac{\pi}{2}\left\|v_{n}\right\|_{1} .
$$

Since $u_{n, m}$ is uniformly bounded in $m$, this implies that $\left\{u_{n, m}\right\}_{m}$ is uniformly bounded in $W^{1,2}(\Omega)$. Then, applying the Minty-Browder's method as in the 
proof of Lemma 3.7 of [2], we can to pass to the limit in (4.2) as $m \rightarrow \infty$ and obtain a function $u_{n} \in W^{1,2}(\Omega)$ such that

$$
\int_{\Omega} w\left(u_{n}-v_{n}\right) d x=-\int_{\Omega} \mathbf{a}_{n}\left(u_{n}, \nabla u_{n}\right) \cdot \nabla w d x \quad \forall w \in W^{1,2}(\Omega),
$$

that is

$$
u_{n}-\operatorname{div} \mathbf{a}_{n}\left(u_{n}, \nabla u_{n}\right)=v_{n} \quad \text { in } \mathcal{D}^{\prime}(\Omega)
$$

$$
\left[\mathbf{a}_{n}\left(u_{n}, \nabla u_{n}\right), v\right]=0 \quad \text { on } \partial \Omega .
$$

Moreover we have the estimates

$$
\left\|u_{n}\right\|_{\infty} \leq\left\|v_{n}\right\|_{\infty} \quad \text { for all } n \in \mathbb{N},
$$

and

$$
\frac{1}{n} \int_{\Omega}\left|\nabla \beta\left(u_{n}\right)\right|^{2} \leq \frac{\pi}{2}\left\|v_{n}\right\|_{1}
$$

Let us prove that $u_{n}$ is a equi-integrable in $L^{1}(\Omega)$. Let

$$
\mathcal{P}_{0}:=\left\{p \in C^{\infty}(\mathbb{R}): 0 \leq p^{\prime} \leq 1, \operatorname{supp}\left(p^{\prime}\right) \text { compact, } 0 \notin \operatorname{supp}(p)\right\} .
$$

Let $p \in \mathcal{P}_{0}$. Multiplying (4.7) by $p\left(u_{n}\right)$, and using Green's formula, we obtain

$$
\begin{aligned}
\int_{\Omega} u_{n} p\left(u_{n}\right) d x & +\int_{\Omega} \mathbf{a}\left(u_{n}, \nabla p\left(u_{n}\right)\right) \cdot \nabla p\left(u_{n}\right) d x+\frac{1}{n} \int_{\Omega} \nabla \beta\left(u_{n}\right) \cdot \nabla p\left(u_{n}\right) d x \\
& =\int_{\Omega} v_{n} p\left(u_{n}\right) d x .
\end{aligned}
$$

Since the second and third integrals of the left hand side of the above identity are positive, we obtain

$$
\int_{\Omega} u_{n} p\left(u_{n}\right) d x \leq \int_{\Omega} v_{n} p\left(u_{n}\right) d x .
$$

This inequality implies that $u_{n}$ is equi-integrable in $L^{1}(\Omega)$. Indeed, it is well known (c.f., e.g., [25]) that there exists an even strictly convex function $M$ : $\mathbb{R} \rightarrow\left[0,+\infty\left[\right.\right.$, with $M(0)=M^{\prime}(0)=0, M$ coercive $\left(\lim _{r \rightarrow+\infty} \frac{M(r)}{r}=+\infty\right)$ and verifying the $\Delta_{2}$-condition, such that $v$ and the sequence $v_{n}$ can be considered as functions in the Orliz space $L_{M}(\Omega)$ contained in $L^{1}(\Omega)$. Then, by results in [10], (4.10) implies that the sequence $\left\{u_{n}\right\}$ is bounded in $L_{M}(\Omega)$. Hence, since $L_{M}(\Omega)$ is reflexive, $\left\{u_{n}\right\}$ is equi-integrable. 
Let us prove estimates on the gradient independent of $n$. For that, we multiply (4.7) by $T\left(u_{n}\right), T=T_{a, b}, a<b$, to obtain, after integration by parts, that

$$
\int_{\Omega} u_{n} T\left(u_{n}\right)+\int_{\Omega} \mathbf{a}\left(u_{n}, \nabla u_{n}\right) \cdot \nabla T\left(u_{n}\right)+\frac{1}{n} \int_{\Omega} \nabla \beta\left(u_{n}\right) \cdot \nabla T\left(u_{n}\right)=\int_{\Omega} v_{n} T\left(u_{n}\right) .
$$

Using (3.7) we obtain

$$
C \int_{\Omega}\left|\nabla T\left(u_{n}\right)\right| \leq\|T\|_{\infty}\left\|v_{n}\right\|_{1}+D|\Omega|
$$

for some constants $C, D>0$ depending on $T$.

Thus, by extracting a subsequence, if necessary, we may assume that $u_{n}$ converges weakly in $L^{1}(\Omega)$ and almost everywhere to some $u \in T B V(\Omega)$ as $n \rightarrow+\infty$. Hence, we have that $u_{n}$ converges to $u$ strongly in $L^{1}(\Omega)$ (in particular, $T\left(u_{n}\right)$ converges in $L^{1}(\Omega)$ to $T(u)$ for any $\left.T=T_{a, b}\right)$.

If (3.5) holds for any $(z, \xi) \in \mathbb{R} \times \mathbb{R}^{N}$, using (4.9) we obtain that $\left\{\mathbf{a}_{n}\left(u_{n}, \nabla u_{n}\right): n \in \mathbb{N}\right\}$ is bounded in $L^{2}\left(\Omega, \mathbb{R}^{N}\right)$. Under the condition (3.5), we assume that $v \in L^{\infty}(\Omega)$, and we take $v_{n}=v$. In that case, $\left\|u_{n}\right\|_{\infty} \leq\|v\|_{\infty}$, and by (3.5) and (4.9), we obtain that $\left\{\mathbf{a}_{n}\left(u_{n}, \nabla u_{n}\right): n \in \mathbb{N}\right\}$ is bounded in $L^{2}\left(\Omega, \mathbb{R}^{N}\right)$. Consequently we may assume that

$$
\mathbf{a}_{n}\left(u_{n}, \nabla u_{n}\right) \rightarrow \mathbf{z} \text { as } n \rightarrow \infty \text {, weakly in } L^{2}\left(\Omega, \mathbb{R}^{N}\right) .
$$

Given $\phi \in C_{0}^{\infty}(\Omega)$, multiplying (4.7) by $\phi$ and integrating by parts, we obtain

$$
\int_{\Omega} \phi\left(v_{n}-u_{n}\right) d x=\int_{\Omega} \mathbf{a}_{n}\left(u_{n}, \nabla u_{n}\right) \cdot \nabla \phi d x .
$$

Letting $n \rightarrow+\infty$, we obtain

$$
\int_{\Omega}(v-u) \phi d x=\int_{\Omega} \mathbf{z} \cdot \nabla \phi d x
$$

that is,

$$
v-u=-\operatorname{div}(\mathbf{z}) \quad \text { in } \quad \mathcal{D}^{\prime}(\Omega)
$$

and

$$
\operatorname{div} \mathbf{a}_{n}\left(u_{n}, \nabla u_{n}\right) \rightarrow \operatorname{div}(\mathbf{z}) \quad \text { in } \quad L^{1}(\Omega) .
$$

Since, by (4.9),

$$
\frac{1}{n}\left|\nabla \beta\left(u_{n}\right)\right| \rightarrow 0 \quad \text { in } \quad L^{2}(\Omega),
$$


as a consequence of (4.11), it follows that

$$
\mathbf{a}\left(u_{n}, \nabla u_{n}\right) \rightarrow \mathbf{z} \text { as } n \rightarrow \infty \text {, weakly in } L^{2}\left(\Omega, \mathbb{R}^{N}\right) .
$$

Moreover, in any of the considered cases we may assume that

$$
\mathbf{a}\left(u_{n}, \nabla u_{n}\right) \rightarrow \mathbf{z} \text { as } n \rightarrow \infty \text {, weakly* in } L^{\infty}\left(\Omega, \mathbb{R}^{N}\right)
$$

By (4.12), we have $\mathbf{z} \in X_{1}(\Omega)$. On the other hand, taking into account (4.7), by (4.11) and (4.13), we obtain that

$$
[\mathbf{z}, v]=0 \quad \mathcal{H}^{N-1}-\text { a.e. on } \partial \Omega
$$

Let us prove that

$$
\mathbf{z}(x)=\mathbf{a}(u(x), \nabla u(x)) \quad \text { a.e. } \quad x \in \Omega .
$$

Let $0 \leq \phi \in C_{0}^{1}(\Omega), \quad g \in C^{2}(\Omega) \cap W^{1, \infty}(\Omega), T=T_{a, b}, a<b$ (in case that $v \in L^{\infty}(\Omega)$ we could dismiss the use of the truncatures $\left.T_{a, b}\right)$. For simplicity, we write $T^{\prime}(r)$ to mean $\chi_{(a, b)}(r)$. By (3.4), we have

$$
\left.\int_{\Omega} \phi\left[\mathbf{a}\left(u_{n}, \nabla u_{n}\right)-\mathbf{a}\left(u_{n}, \nabla g\right)\right) \cdot \nabla\left(u_{n}-g\right)\right] T^{\prime}\left(u_{n}\right) d x \geq 0 .
$$

Now, since

$$
\begin{aligned}
& \int_{\Omega} \phi \mathbf{a}\left(u_{n}, \nabla u_{n}\right) \cdot \nabla\left(u_{n}-g\right) T^{\prime}\left(u_{n}\right) d x \\
& =\int_{\Omega} \phi \mathbf{a}\left(u_{n}, \nabla u_{n}\right) \cdot \nabla\left(T\left(u_{n}\right)-g\right) d x+\int_{\Omega} \phi \mathbf{a}\left(u_{n}, \nabla u_{n}\right) \cdot \nabla g\left(1-T^{\prime}\left(u_{n}\right)\right) d x \\
& =\int_{\Omega} \phi \mathbf{a}_{n}\left(u_{n}, \nabla u_{n}\right) \cdot \nabla\left(T\left(u_{n}\right)-g\right) d x-\frac{1}{n} \int_{\Omega} \phi \nabla \beta\left(u_{n}\right) \cdot \nabla\left(T\left(u_{n}\right)-g\right) d x \\
& \quad+\int_{\Omega} \phi \mathbf{a}\left(u_{n}, \nabla u_{n}\right) \cdot \nabla g\left(1-T^{\prime}\left(u_{n}\right)\right) d x \\
& \leq-\int_{\Omega} \operatorname{div}\left(\mathbf{a}_{n}\left(u_{n}, \nabla u_{n}\right)\right) \phi\left(T\left(u_{n}\right)-g\right) d x-\int_{\Omega}\left(T\left(u_{n}\right)-g\right) \mathbf{a}_{n}\left(u_{n}, \nabla u_{n}\right) \cdot \nabla \phi d x \\
& \quad+\frac{1}{n} \int_{\Omega} \phi \nabla \beta\left(u_{n}\right) \cdot \nabla g d x+\int_{\Omega} \phi \mathbf{a}\left(u_{n}, \nabla u_{n}\right) \cdot \nabla g\left(1-T^{\prime}\left(u_{n}\right)\right) d x \\
& \leq-\int_{\Omega} \operatorname{div}\left(\mathbf{a}_{n}\left(u_{n}, \nabla u_{n}\right)\right) \phi\left(T\left(u_{n}\right)-g\right) d x-\int_{\Omega}\left(T\left(u_{n}\right)-g\right) \mathbf{a}_{n}\left(u_{n}, \nabla u_{n}\right) \cdot \nabla \phi d x \\
& \quad+\frac{1}{n} \int_{\Omega} \phi \nabla \beta\left(u_{n}\right) \cdot \nabla g d x+M\|\nabla g\|_{\infty} \phi\left[\left(1-T_{\Omega}^{\prime}\left(u_{n}\right)\right) T^{\prime}(u(x))+\left(1-T^{\prime}(u(x))\right)\right] d x
\end{aligned}
$$


we get

$$
\begin{aligned}
& \limsup _{n \rightarrow \infty} \int_{\Omega} \phi \mathbf{a}\left(u_{n}, \nabla u_{n}\right) \cdot \nabla\left(u_{n}-g\right) T^{\prime}\left(u_{n}\right) d x \\
& \leq-\int_{\Omega} \operatorname{div}(\mathbf{z}) \phi(T(u)-g) d x-\int_{\Omega}(T(u)-g) \mathbf{z} \cdot \nabla \phi d x \\
& \quad+M\|\nabla g\|_{\infty} \int_{\Omega} \phi\left(1-T^{\prime}(u)\right) d x \\
& =\int_{\Omega} \phi(\mathbf{z}, D(u-g))+M\|\nabla g\|_{\infty} \int_{\Omega} \phi\left(1-T^{\prime}(u)\right) d x .
\end{aligned}
$$

On the other hand, let us denote by

$$
J_{\mathbf{a}_{i}}(x, r):=\int_{0}^{r} \mathbf{a}_{i}(s, \nabla g(x)) d s, \quad \text { and } \quad J_{\frac{\partial \mathbf{a}_{i}}{\partial x_{j}}}(x, r):=\int_{0}^{r} \frac{\partial}{\partial x_{j}} \mathbf{a}_{i}(s, \nabla g(x)) d s,
$$

$i, j \in\{1, \ldots, N\}$, and observe that, since

$$
\mathbf{a}_{i}\left(T\left(u_{n}(x)\right), \nabla g(x)\right) \frac{\partial u_{n}}{\partial x_{j}}(x) T^{\prime}\left(u_{n}\right)=\mathbf{a}_{i}\left(u_{n}(x), \nabla g(x)\right) \frac{\partial u_{n}}{\partial x_{j}}(x) T^{\prime}\left(u_{n}\right),
$$

we have

$$
\frac{\partial}{\partial x_{j}} J_{\mathbf{a}_{i}}\left(x, T\left(u_{n}(x)\right)\right)=\mathbf{a}_{i}\left(u_{n}(x), \nabla g(x)\right) \frac{\partial u_{n}}{\partial x_{j}}(x) T^{\prime}\left(u_{n}\right)+J_{\frac{\partial \mathbf{a}_{i}}{\partial x_{j}}}\left(x, T\left(u_{n}(x)\right)\right) .
$$

We note that assumption $\left(\mathrm{H}_{2}\right)$ is used here, and, as we shall notice in Remark 4.3, $\left(\mathrm{H}_{2}\right)$ is not needed when $f(z, \xi)$ has the form described in Remark 3.1. Now, since

$$
\frac{\partial}{\partial x_{j}} J_{\mathbf{a}_{i}}\left(x, T\left(u_{n}\right)\right) \rightarrow \frac{\partial}{\partial x_{j}} J_{\mathbf{a}_{i}}(x, T(u))
$$

weakly as measures, and $J_{\frac{\partial \mathbf{a}_{i}}{\partial x_{j}}}\left(x, T\left(u_{n}(x)\right)\right) \rightarrow J_{\frac{\partial \mathbf{a}_{i}}{\partial x_{j}}}(x, T(u(x)))$ a.e., we have

$$
\begin{aligned}
\liminf _{n \rightarrow \infty} \int_{\Omega} \phi \mathbf{a}\left(u_{n}, \nabla g\right) \cdot \nabla\left(u_{n}-g\right) T^{\prime}\left(u_{n}\right) d x \\
=\lim _{n \rightarrow \infty} \int_{\Omega} \phi \sum_{i=1}^{N}\left[\frac{\partial}{\partial x_{i}} J_{\mathbf{a}_{i}}\left(x, T\left(u_{n}(x)\right)\right)-J_{\frac{\partial \mathbf{a}_{i}}{\partial x_{i}}}\left(x, T\left(u_{n}(x)\right)\right)\right] \\
\quad-\limsup _{n \rightarrow \infty} \int_{\Omega} \phi \mathbf{a}\left(u_{n}, \nabla g\right) \cdot \nabla g T^{\prime}\left(u_{n}\right) d x \\
\geq \int_{\Omega} \phi \sum_{i=1}^{N}\left[\frac{\partial}{\partial x_{i}} J_{\mathbf{a}_{i}}(x, T(u))-J_{\frac{\partial \mathbf{a}_{i}}{\partial x_{i}}}(x, T(u(x)))\right] \\
\quad-\int_{\Omega} \phi \mathbf{a}(u, \nabla g) \cdot \nabla g T^{\prime}(u) d x-M\|\nabla g\|_{\infty} \int_{\Omega} \phi\left(1-T^{\prime}(u)\right) d x .
\end{aligned}
$$


Consequently, we obtain

$$
\begin{aligned}
& \int_{\Omega} \phi(\mathbf{z}, D(T(u)-g))+2 M\|g\|_{\infty} \int_{\Omega} \phi\left(1-T^{\prime}(u)\right) \\
& -\int_{\Omega} \phi\left(\sum_{i=1}^{N}\left[\frac{\partial}{\partial x_{i}} J_{\mathbf{a}_{i}}(x, T(u(x)))-J_{\frac{\partial \mathbf{a}_{i}}{\partial x_{i}}}(x, T(u(x)))\right]-\mathbf{a}(u, \nabla g) \cdot \nabla g T^{\prime}(u)\right) \\
& \geq 0,
\end{aligned}
$$

for all $0 \leq \phi \in C_{0}^{1}(\Omega)$. Thus the measure

$$
\begin{aligned}
(\mathbf{z}, D(T(u)-g)) & -\sum_{i=1}^{N}\left[\frac{\partial}{\partial x_{i}} J_{\mathbf{a}_{i}}(x, T(u(x)))-J_{\frac{\partial \mathbf{a}_{i}}{\partial x_{i}}}(x, T(u(x)))\right] \\
& +\mathbf{a}(u, \nabla g) \cdot \nabla g T^{\prime}(u) \mathcal{L}^{N}+2 M\|\nabla g\|_{\infty}\left(1-T^{\prime}(u)\right) \mathcal{L}^{N} \geq 0 .
\end{aligned}
$$

Then using chain's rule for $B V$ functions ([1], Theorem 3.96) applied to $J_{\mathbf{a}_{i}}\left(u_{1}, u_{2}\right)$ with $u_{1}(x)=x, u_{2}(x)=T(u(x)), x \in \Omega$, we deduce that the absolutely continuous part of

$$
\sum_{i=1}^{N}\left[\frac{\partial}{\partial x_{i}} J_{\mathbf{a}_{i}}(x, T(u(x)))-J_{\frac{\partial \mathbf{a}_{i}}{\partial x_{i}}}(x, T(u(x)))\right]
$$

is $\mathbf{a}(u, \nabla g) \cdot \nabla T(u) \mathcal{L}^{N}$ and we obtain

$$
\begin{aligned}
\mathbf{z} \cdot \nabla(T(u)-g) & -\mathbf{a}(u, \nabla g) \cdot \nabla T(u)+\mathbf{a}(u, \nabla g) \cdot \nabla g T^{\prime}(u) \\
& +2 M\|\nabla g\|_{\infty}\left(1-T^{\prime}(u)\right) \geq 0 \quad \text { a.e. }
\end{aligned}
$$

In particular, we deduce that

$$
(\mathbf{z}-\mathbf{a}(u, \nabla g)) \cdot \nabla(u-g) \geq 0 \quad \text { a.e. in }[a<u<b] .
$$

Since this holds for any values of $a, b, a<b$, we have that the above inequality holds a.e. in $\Omega$. Since we may take a countable set of functions $g \in C^{2}(\Omega) \cap$ $W^{1, \infty}(\Omega)$ dense in $C^{1}(\bar{\Omega})$ we have that the above inequality holds for all $x \in \tilde{\Omega}$, where $\tilde{\Omega} \subset \Omega$ is such that $\mathcal{L}^{N}(\Omega \backslash \tilde{\Omega})=0$, and all $g \in C^{1}(\bar{\Omega})$. Now, fixed $x \in \tilde{\Omega}$ and given $\xi \in \mathbb{R}^{N}$, there is $g \in C^{1}(\bar{\Omega})$ such that $\nabla g(x)=\xi$. Then

$$
(\mathbf{z}(x)-\mathbf{a}(u(x), \xi)) \cdot(\nabla u(x)-\xi) \geq 0, \quad \forall \xi \in R^{N} .
$$

These inequalities imply (4.18) by an application of Minty-Browder's method in $\mathbb{R}^{N}$.

From (4.18), (4.12) and (4.17), it follows that

$$
v-u=-\operatorname{div} \mathbf{a}(u, \nabla u), \quad \text { in } \mathcal{D}^{\prime}(\Omega)
$$


and

$$
[\mathbf{a}(u, \nabla u), v]=0 \quad \mathcal{H}^{N-1}-\text { a.e. on } \partial \Omega \text {. }
$$

Therefore, to finish the existence part of the proof we only need to prove that

$$
(\mathbf{a}(u, \nabla u), D T(u)) \geq h(u, D T(u)) \quad \text { as measures } \forall T=T_{a, b}, a<b .
$$

To do that, first let us prove

$$
\underset{n}{\lim \sup _{\Omega}} \int_{\Omega} \mathbf{a}\left(u_{n}, \nabla u_{n}\right) \cdot \nabla T\left(u_{n}\right) \phi(x) d x \leq \int_{\Omega} \phi(\mathbf{a}(u, \nabla u), D T(u))
$$

for any $0 \leq \phi \in \mathcal{D}(\Omega)$. For that, if we take $w=T\left(u_{n}\right) \phi$ as test function in (4.6), we obtain

$$
\begin{aligned}
\int_{\Omega} \phi \mathbf{a}\left(u_{n}, \nabla u_{n}\right) \cdot \nabla T\left(u_{n}\right) d x+\frac{1}{n} \int_{\Omega} \phi \nabla \beta\left(u_{n}\right) \cdot \nabla T\left(u_{n}\right) d x \\
=\int_{\Omega}\left(v_{n}-u_{n}\right) T\left(u_{n}\right) \phi d x-\int_{\Omega} T\left(u_{n}\right) \mathbf{a}\left(u_{n}, \nabla u_{n}\right) \cdot \nabla \phi d x \\
\quad-\frac{1}{n} \int_{\Omega} T\left(u_{n}\right) \nabla \beta\left(u_{n}\right) \cdot \nabla \phi d x .
\end{aligned}
$$

Since the sign of the second term at the left hand side of the above inequality is positive, letting $n \rightarrow \infty$ we get

$$
\begin{aligned}
\limsup _{n} & \int_{\Omega} \phi \mathbf{a}\left(u_{n}, \nabla u_{n}\right) \cdot \nabla T\left(u_{n}\right) d x \\
& \leq \int_{\Omega} \phi(v-u) T(u) d x-\int_{\Omega} T(u) \mathbf{a}(u, \nabla u) \cdot \nabla \phi d x \\
& =-\int_{\Omega} \operatorname{div} \mathbf{a}(u, \nabla u) T(u) \phi d x \\
& -\int_{\Omega} T(u) \mathbf{a}(u, \nabla u) \cdot \nabla \phi=\int_{\Omega} \phi(\mathbf{a}(u, \nabla u), D T(u)) .
\end{aligned}
$$

Now, let us prove the following inequality for measures

$$
f(T(u), D T(u)) \leq(\mathbf{a}(u, \nabla u), D T(u))+f(T(u), 0) .
$$

Using the convexity of $f$, and observing that

$$
\mathbf{a}\left(T\left(u_{n}\right), \nabla T\left(u_{n}\right)\right) \cdot \nabla T\left(u_{n}\right)=\mathbf{a}\left(u_{n}, \nabla T\left(u_{n}\right)\right) \cdot \nabla T\left(u_{n}\right),
$$


we have for any $w \in W^{1,1}(\Omega)$

$$
\begin{aligned}
\int_{\Omega} \phi f\left(T\left(u_{n}\right), \nabla T\left(u_{n}\right)\right) d x \leq & \int_{\Omega} \phi \mathbf{a}\left(u_{n}, \nabla T\left(u_{n}\right)\right) \cdot \nabla T\left(u_{n}\right) d x \\
& -\int_{\Omega} \phi \mathbf{a}\left(T\left(u_{n}\right), \nabla T\left(u_{n}\right)\right) \cdot \nabla w d x \\
& +\int_{\Omega} f\left(T\left(u_{n}\right), \nabla w\right) \phi d x
\end{aligned}
$$

Choosing $w=0$, we obtain

$$
\begin{aligned}
\int_{\Omega} \phi f\left(T\left(u_{n}\right), \nabla T\left(u_{n}\right)\right) d x \leq & \int_{\Omega} \phi \mathbf{a}\left(u_{n}, \nabla T\left(u_{n}\right)\right) \cdot \nabla T\left(u_{n}\right) d x \\
& +\int_{\Omega} \phi f\left(T\left(u_{n}\right), 0\right) d x .
\end{aligned}
$$

By Proposition 2.2 and (4.23), letting $n \rightarrow \infty$ we obtain

$$
\begin{aligned}
\mathcal{R}_{f}^{a, b}(T(u), \phi) & \leq \liminf _{n} \int_{\Omega} f\left(T\left(u_{n}\right), \nabla T\left(u_{n}\right)\right) \phi d x \\
& \leq \liminf _{n} \int_{\Omega} \phi \mathbf{a}\left(u_{n}, \nabla T\left(u_{n}\right)\right) \cdot \nabla T\left(u_{n}\right) d x+\int_{\Omega} f(T(u), 0) \phi d x \\
& \leq \int_{\Omega} \phi(\mathbf{a}(u, \nabla u), D T(u))+\int_{\Omega} \phi f(T(u), 0) d x
\end{aligned}
$$

and (4.24) holds.

Note that by the definition of $\mathcal{R}_{f}^{a, b}(u, \phi)$, using the chain rule for $\mathrm{BV}$ functions, we deduce that

$$
f(T(u), D T(u))^{s}=\psi^{0}\left(\frac{D T(u)}{|D T(u)|}\right)\left|D^{s} J_{\varphi}(T(u))\right| .
$$

Then using (4.24), we have

$$
\begin{aligned}
& (\mathbf{a}(u, \nabla u), D T(u))=(\mathbf{a}(u, \nabla u), D T(u))^{a c}+(\mathbf{a}(u, \nabla u), D T(u))^{s} \\
& \geq a(u, \nabla u) \cdot \nabla T(u)+\psi^{0}\left(\frac{D T(u)}{|D T(u)|}\right)\left|D^{s} J_{\varphi}(T(u))\right|=h(u, D T(u)) .
\end{aligned}
$$

Step 2. Uniqueness of entropy solutions. Given $v, \bar{v} \in L^{1}(\Omega)$, let $u, \bar{u} \in T B V(\Omega)$ be two entropy solutions of the problems

$$
\begin{cases}u-\operatorname{div} \mathbf{a}(u, D u)=v & \text { in } \Omega \\ \frac{\partial u}{\partial \eta}=0 & \text { on } \partial \Omega\end{cases}
$$


and

$$
\begin{cases}\bar{u}-\operatorname{div} \mathbf{a}(\bar{u}, D \bar{u})=\bar{v} & \text { in } \Omega \\ \frac{\partial \bar{u}}{\partial \eta}=0 & \text { on } \partial \Omega\end{cases}
$$

respectively. Let us assume that $\bar{v} \in L^{\infty}(\Omega)$. In this case, also $\bar{u} \in L^{\infty}(\Omega)$. Indeed, to prove this observation it suffices to multiply (4.26) by $T_{0, \varepsilon}\left(\bar{u}-\|\bar{v}\|_{\infty}\right)$, and, integrating by parts, we obtain

$$
\int_{\Omega} T_{0, \varepsilon}\left(\bar{u}-\|\bar{v}\|_{\infty}\right)(\bar{u}-\bar{v}) d x \leq 0
$$

Now, dividing by $\varepsilon$ and letting $\varepsilon \rightarrow 0+$, we obtain

$$
0 \leq \int_{\bar{u}>\|\bar{v}\|_{\infty}}(\bar{u}-\bar{v}) d x \leq 0 .
$$

We deduce that $\left|\left\{x \in \Omega: \bar{u}>\|\bar{v}\|_{\infty}\right\}\right|=0$, i.e., $\bar{u} \leq\|\bar{v}\|_{\infty}$. In a similar way we prove that $-\|\bar{v}\|_{\infty} \leq \bar{u}$.

Let $\rho_{n}$ be a classical mollifiers in $\mathbb{R}^{N}$, and let us write $\xi_{n}(x, y)=\rho_{n}(x-y)$. If we denote $\mathbf{z}(y)=\mathbf{a}(u(x), \nabla u(x))$ and $\overline{\mathbf{z}}(x)=\mathbf{a}(\bar{u}(x), \nabla \bar{u}(y))$, we have

$$
u-\operatorname{div}(\mathbf{z})=v \quad \text { and } \quad \bar{u}-\operatorname{div}(\overline{\mathbf{z}})=\bar{v} \quad \text { in } \quad \mathcal{D}^{\prime}(\Omega)
$$

Then, multiplying both equations by $T_{\varepsilon}(u(y)-\bar{u}(x)) \xi_{n}(x, y)$ and integrating by parts, we obtain

$$
\begin{aligned}
& \int_{\Omega} u(y) T_{\varepsilon}(u(y)-\bar{u}(x)) \xi_{n}(x, y) d y+\int_{\Omega} \xi_{n}\left(\mathbf{z}, D_{y}\left(T_{\varepsilon}(u-\bar{u}(x))\right.\right. \\
& \quad+\int_{\Omega} T_{\varepsilon}(u(y)-\bar{u}(x)) \mathbf{z}(y) \cdot \nabla_{y} \xi_{n}(x, y) d y \\
& =\int_{\Omega} v(y) T_{\varepsilon}(u(y)-\bar{u}(x)) \xi_{n}(x, y) d y
\end{aligned}
$$

and

$$
\begin{aligned}
& \int_{\Omega} \bar{u}(x) T_{\varepsilon}(u(y)-\bar{u}(x)) \xi_{n}(x, y) d x+\int_{\Omega} \xi_{n}\left(\overline{\mathbf{z}}, D_{x}\left(T_{\varepsilon}(u(y)-\bar{u})\right)\right. \\
& \quad+\int_{\Omega} T_{\varepsilon}(u(y)-\bar{u}(x)) \bar{z}(x) \cdot \nabla_{x} \xi_{n}(x, y) d x \\
& =\int_{\Omega} \bar{v}(x) T_{\varepsilon}(u(y)-\bar{u}(x)) \xi_{n}(x, y) d x .
\end{aligned}
$$


Integrating (4.27) in $x$ and (4.28) in $y$, and taking differences we obtain

$$
\begin{aligned}
\int_{\Omega} \int_{\Omega}(u(y)- & \bar{u}(x)) T_{\varepsilon}(u(y)-\bar{u}(x)) \xi_{n}(x, y) d x d y \\
& +\int_{\Omega}\left(\int_{\Omega} \xi_{n}\left(\mathbf{z}, D_{y}\left(T_{\varepsilon}(u-\bar{u}(x))\right)\right) d x\right. \\
& +\int_{\Omega \times \Omega} T_{\varepsilon}(u(y)-\bar{u}(x)) \mathbf{z}(y) \cdot \nabla_{y} \xi_{n}(x, y) d y d x \\
& -\int_{\Omega}\left(\int _ { \Omega } \xi _ { n } \left(\overline{\mathbf{z}}, D_{x}\left(T_{\varepsilon}(u(y)-\bar{u})\right) d y\right.\right. \\
& -\int_{\Omega \times \Omega} T_{\varepsilon}(u(y)-\bar{u}(x)) \overline{\mathbf{z}}(x) \cdot \nabla_{x} \xi_{n}(x, y) d x d y \\
= & \int_{\Omega} \int_{\Omega}(v(y)-\bar{v}(x)) T_{\varepsilon}(u(y)-\bar{u}(x)) \xi_{n}(x, y) d x d y .
\end{aligned}
$$

Let $I_{1}^{n}, I_{2}^{n}$ be, respectively, the first term at the rest of the terms at the left hand side of the above identity, and let $I_{3}^{n}$ be the right hand side term. Note that, by applying Green's formula and using that $T_{\varepsilon}(-r)=-T_{\varepsilon}(r)$ and $\nabla_{y} \xi_{n}(x, y)+$ $\nabla_{x} \xi_{n}(x, y)=0$, we get

$$
\begin{aligned}
I_{2}^{n}= & \int_{\Omega}\left(\int_{\Omega} \xi_{n}\left(\mathbf{z}, D_{y}\left(T_{\varepsilon}(u-\bar{u}(x))\right)\right) d x-\int_{\Omega}\left(\int_{\Omega} \xi_{n}\left(\overline{\mathbf{z}}, D_{x}\left(T_{\varepsilon}(u(y)-\bar{u})\right)\right)\right) d y\right. \\
& +\int_{\Omega \times \Omega} T_{\varepsilon}(u(y)-\bar{u}(x)) \mathbf{z}(y) \cdot \nabla_{y} \xi_{n}(x, y) d y d x \\
& -\int_{\Omega \times \Omega} T_{\varepsilon}(u(y)-\bar{u}(x)) \overline{\mathbf{z}}(x) \cdot \nabla_{x} \xi_{n}(x, y) d x d y \\
= & \int_{\Omega \times \Omega} T_{\varepsilon}(u(y)-\bar{u}(x))\left[(\mathbf{z}(y)-\overline{\mathbf{z}}(x)) \cdot\left(\nabla_{y} \xi_{n}(x, y)+\nabla_{x} \xi_{n}(x, y)\right)\right] d y d x \\
& +\int_{\Omega}\left(\int_{\Omega} \xi_{n}\left(\mathbf{z}, D_{y}\left(T_{\varepsilon}(u-\bar{u}(x))\right)\right) d x\right. \\
& +\int_{\Omega \times \Omega} T_{\varepsilon}(u(y)-\bar{u}(x)) \overline{\mathbf{z}}(x) \cdot \nabla_{y} \xi_{n}(x, y) d x d y \\
& -\int_{\Omega}\left(\int_{\Omega} \xi_{n}\left(\overline{\mathbf{z}}, D_{x}\left(T_{\varepsilon}(u(y)-\bar{u})\right)\right)\right) d y \\
& -\int_{\Omega \times \Omega} T_{\varepsilon}(u(y)-\bar{u}(x)) \mathbf{z}(y) \cdot \nabla_{x} \xi_{n}(x, y) d y d x \\
= & \int_{\Omega}\left(\int_{\Omega} \xi_{n}\left(\mathbf{z}, D_{y}\left(T_{\varepsilon}(u-\bar{u}(x))\right)\right) d x-\int_{\Omega}\left(\int_{\Omega} \xi_{n}\left(\overline{\mathbf{z}}(x), D_{y} T_{\varepsilon}(u-\bar{u}(x))\right) d x\right.\right. \\
& +\int_{\Omega}\left(\int_{\Omega} \xi_{n}\left(\overline{\mathbf{z}}, D_{x}\left(T_{\varepsilon}(\bar{u}-u(y))\right)\right)\right) d y-\int_{\Omega}\left(\int_{\Omega} \xi_{n}\left(\mathbf{z}(y), D_{x} T_{\varepsilon}(\bar{u}-u(y))\right)\right) d y .
\end{aligned}
$$

Let us define $\bar{u}_{\varepsilon}(x, y):=T_{-\varepsilon+u(y), \varepsilon+u(y)}(\bar{u}(x)), u_{\varepsilon}(y, x):=T_{-\varepsilon+\bar{u}(x), \varepsilon+\bar{u}(x)}(u(y))$. Observe that, since $\bar{u} \in L^{\infty}(\Omega)$, we have that $\bar{u}_{\varepsilon}(\cdot, y), u_{\varepsilon}(x, \cdot) \in B V(\Omega)$. Since

$$
\left.T_{\varepsilon}(\bar{u}(x)-u(y))=\bar{u}_{\varepsilon}(x, y)-u(y), \quad T_{\varepsilon}(u-\bar{u}(x))=u_{\varepsilon}(y, x)-\bar{u}(x)\right)
$$


we have

$$
D_{x}\left(T_{\varepsilon}(\bar{u}-u(y))\right)=D_{x} \bar{u}_{\varepsilon}(\cdot, y), \quad D_{y}\left(T_{\varepsilon}(u-\bar{u}(x))\right)=D_{y} u_{\varepsilon}(\cdot, x) .
$$

Therefore, we can write

$$
\begin{aligned}
I_{2}^{n}= & \int_{\Omega}\left(\int_{\Omega} \xi_{n}(x, y) \mathbf{z}(y) \cdot \nabla_{y} u_{\varepsilon}(y, x) d y\right) d x \\
& -\int_{\Omega}\left(\int_{\Omega} \xi_{n}(x, y) \overline{\mathbf{z}}(x) \cdot \nabla_{y} u_{\varepsilon}(y, x) d y\right) d x \\
& +\int_{\Omega}\left(\int_{\Omega} \xi_{n} \mathbf{z} \cdot D_{y}^{s} u_{\varepsilon}(\cdot, x)\right) d x-\int_{\Omega}\left(\int_{\Omega} \xi_{n} \overline{\mathbf{z}}(x) \cdot D_{y}^{s} u_{\varepsilon}(\cdot, x)\right) d x \\
& +\int_{\Omega}\left(\int_{\Omega} \xi_{n}(x, y) \overline{\mathbf{z}}(x) \cdot \nabla_{x} \bar{u}_{\varepsilon}(x, y) d x\right) d y \\
& -\int_{\Omega}\left(\int_{\Omega} \xi_{n}(x, y) \mathbf{z}(y) \cdot \nabla_{x} \bar{u}_{\varepsilon}(x, y) d x\right) d y \\
& +\int_{\Omega}\left(\int_{\Omega} \xi_{n} \overline{\mathbf{z}} \cdot D_{x}^{s} \bar{u}_{\varepsilon}(\cdot, y) d x\right) d y-\int_{\Omega}\left(\int_{\Omega} \xi_{n} \mathbf{z}(y) \cdot D_{x}^{s} \bar{u}_{\varepsilon}(\cdot, y) d x\right) d y \\
= & \int_{\Omega \times \Omega} \xi_{n}(x, y)(\mathbf{z}(y)-\overline{\mathbf{z}}(x)) \cdot\left(\nabla_{y} u_{\varepsilon}(y, x)-\nabla_{x} \bar{u}_{\varepsilon}(x, y)\right) d y d x \\
& +\int_{\Omega}\left(\int_{\Omega} \xi_{n} \overline{\mathbf{z}} \cdot D_{x}^{s} \bar{u}_{\varepsilon}(\cdot, y) d x\right) d y-\int_{\Omega}\left(\int_{\Omega} \xi_{n} \mathbf{z}(y) \cdot D_{x}^{s} \bar{u}_{\varepsilon}(\cdot, y) d x\right) d y \\
& +\int_{\Omega}\left(\int_{\Omega} \xi_{n} \mathbf{z} \cdot D_{y}^{s} u_{\varepsilon}(\cdot, x)\right) d x-\int_{\Omega}\left(\int_{\Omega} \xi_{n} \overline{\mathbf{z}}(x) \cdot D_{y}^{s} u_{\varepsilon}(\cdot, x)\right) d x \\
= & I_{21}^{n}+I_{22}^{n}+I_{23}^{n} .
\end{aligned}
$$

Let us compute $I_{21}^{n}$. Let us write $S=T_{-\|\bar{u}\|_{\infty}-\varepsilon,\|\bar{u}\|_{\infty}+\varepsilon}$. By (3.9) it follows that

$$
\begin{aligned}
I_{21}^{n} & =\int_{\Omega \times \Omega} \xi_{n}(x, y)(\mathbf{z}(y)-\overline{\mathbf{z}}(x)) \cdot\left(\nabla_{y} u_{\varepsilon}(y, x)-\nabla_{x} \bar{u}_{\varepsilon}(x, y)\right) d y d x \\
& =\int_{\Omega \times \Omega} \xi_{n}(x, y) T_{\varepsilon}^{\prime}(u(y)-\bar{u}(x))(\mathbf{z}(y)-\overline{\mathbf{z}}(x)) \cdot\left(\nabla_{y} u(y)-\nabla_{x} \bar{u}(x)\right) d y d x \\
& =\int_{\Omega \times \Omega} \xi_{n}(x, y) T_{\varepsilon}^{\prime}(u(y)-\bar{u}(x))(\mathbf{z}(y)-\overline{\mathbf{z}}(x)) \cdot\left(\nabla_{y} S(u(y))-\nabla_{x} \bar{u}(x)\right) d y d x \\
& \geq-C \int_{\Omega \times \Omega} \xi_{n}(x, y) T_{\varepsilon}^{\prime}(u(y)-\bar{u}(x))|u(y)-\bar{u}(x)|\left\|\nabla_{y} S(u(y))-\nabla_{x} \bar{u}(x)\right\| d y d x \\
& \geq-C \varepsilon \int_{\Omega}\left(\int_{u(y)-\varepsilon}^{u(y)+\varepsilon} \xi_{n}(x, y)\left\|\nabla_{y} S(u(y))-\nabla_{x} \bar{u}(x)\right\| d x\right) d y .
\end{aligned}
$$

Hence

$$
\frac{1}{\varepsilon} I_{21}^{n} \geq o(\varepsilon) \quad \forall n \in \mathbb{N}
$$

where $o(\varepsilon)$ is an expression that tends to 0 as $\varepsilon \rightarrow 0^{+}$. 
Now we analyze $I_{22}^{n}$. Having in mind condition (3.13) of the definition of entropy solution, we obtain that

$$
\int_{\Omega}\left(\int_{\Omega} \xi_{n} \overline{\mathbf{z}} \cdot D_{x}^{s} \bar{u}_{\varepsilon}(\cdot, y) d x\right) d y \geq \int_{\Omega}\left(\int_{\Omega} \xi_{n} h\left(\bar{u}_{\varepsilon}(\cdot, y), D_{x} \bar{u}_{\varepsilon}(\cdot, y)\right)^{s}\right) d y
$$

On the other hand, by $\left(\mathrm{H}_{5}\right)$ and $\left(\mathrm{H}_{6}\right)$, we have

$$
\mathbf{z}(y) \cdot D_{x}^{s} \bar{u}_{\varepsilon}(\cdot, y)=\left(\mathbf{z}(y) \cdot \vec{D}^{s} \bar{u}_{\varepsilon}\right)\left|D_{x}^{s} \bar{u}_{\varepsilon}\right| \leq \varphi(u(y)) \psi^{0}\left(\overrightarrow{D^{s} \bar{u}_{\varepsilon}}\right)\left|D_{x}^{s} \bar{u}_{\varepsilon}\right| .
$$

Therefore we have

$$
\begin{aligned}
I_{22}^{n} \geq & \int_{\Omega}\left(\int_{\Omega} \xi_{n} h\left(\bar{u}_{\varepsilon}, D_{x} \bar{u}_{\varepsilon}\right)^{s}\right) d y-\int_{\Omega}\left(\int_{\Omega} \xi_{n} \varphi(u(y)) \psi^{0}\left({\overrightarrow{D^{s} \bar{u}_{\varepsilon}}}\right)\left|D_{x}^{s} \bar{u}_{\varepsilon}\right|\right) d y \\
= & \int_{\Omega}\left(\int_{\Omega} \xi_{n} \varphi\left(\bar{u}_{\varepsilon}(x)\right) \psi^{0}\left({\overrightarrow{D^{s} \bar{u}_{\varepsilon}}}\right)\left|D_{x}^{c} \bar{u}_{\varepsilon}\right|\right) d y \\
& -\int_{\Omega}\left(\int_{\Omega} \xi_{n} \varphi(u(y)) \psi^{0}\left({\overrightarrow{D^{s} \bar{u}_{\varepsilon}}}\right)\left|D_{x}^{c} \bar{u}_{\varepsilon}\right|\right) d y \\
& +\int_{\Omega}\left(\int_{J_{\bar{u}_{\varepsilon}}} \xi_{n} \frac{1}{\left(\bar{u}_{\varepsilon}\right)^{+}(x)-\left(\bar{u}_{\varepsilon}\right)^{-}(x)}\left(\int_{\left(\bar{u}_{\varepsilon}\right)^{-(x)}}^{\left(\bar{u}_{\varepsilon}\right)^{+}(x)} \varphi(s) d s\right) \psi^{0}\left(\vec{D}^{s} \vec{u}_{\varepsilon}\right)\left|D_{x}^{j} \bar{u}_{\varepsilon}\right|\right) d y \\
& -\int_{\Omega}\left(\int_{\Omega} \xi_{n} \varphi(u(y)) \psi^{0}\left({\overrightarrow{D^{s} \bar{u}_{\varepsilon}}}\right)\left|D_{x}^{j} \bar{u}_{\varepsilon}\right|\right) d y=J_{1}^{n}+J_{2}^{n}
\end{aligned}
$$

where $J_{1}^{n}$ denotes the first and second terms of the above expression, and $J_{2}^{n}$ the third and fourth terms. Now, since $\varphi$ is Lipschitz continuous, we have

$$
\begin{aligned}
\left|J_{1}^{n}\right| & \leq \int_{\Omega}\left(\int_{\Omega} \xi_{n}\left|\varphi\left(\bar{u}_{\varepsilon}(x)\right)-\varphi(u(y))\right| \psi^{0}\left(\overrightarrow{D^{s} \bar{u}_{\varepsilon}}\right)\left|D_{x}^{c} \bar{u}_{\varepsilon}\right|\right) d y \\
& \leq M_{n} \int_{\Omega}\left(\int_{\Omega}\left|\bar{u}_{\varepsilon}(x)-u(y)\right| T_{-\varepsilon+u(y), \varepsilon+u(y)}^{\prime}(\bar{u}(x))\left|D_{x}^{c} \bar{u}\right|\right) d y \\
& =M_{n} \int_{\Omega}\left(\int_{\{x \in \Omega:-\varepsilon+u(y)<\bar{u}(x)<\varepsilon+u(y)\}}\left|\bar{u}_{\varepsilon}(x)-u(y)\right|\left|D_{x}^{c} \bar{u}\right|\right) d y \\
& \leq 2 \varepsilon M_{n} \int_{\Omega}\left(\int_{\Omega}\left|D_{x}^{c} \bar{u}_{\varepsilon}\right|\right) .
\end{aligned}
$$

Using the coarea formula, we get

$$
\left|J_{1}^{n}\right| \leq 2 \varepsilon M_{n} \int_{\Omega}\left(\int_{u(y)-\varepsilon}^{u(y)+\varepsilon} \operatorname{Per}(\{\bar{u}(x) \geq \lambda\}) d \lambda\right) d y
$$

which yields

$$
\lim _{\varepsilon \rightarrow 0^{+}} \frac{1}{\varepsilon} J_{1}^{n}=0 \quad \forall n \in \mathbb{N}
$$


On the other hand, working in a similar way as before, we have

$$
\begin{aligned}
\left|J_{2}^{n}\right| & \leq \int_{\Omega}\left[\int_{\bar{u}_{\bar{\varepsilon}}} \xi_{n} \frac{1}{\left(\bar{u}_{\varepsilon}\right)^{+}(x)-\left(\bar{u}_{\varepsilon}\right)^{-}(x)}\left(\int_{\left(\bar{u}_{\varepsilon}\right)^{-}(x)}^{\left(\bar{u}_{\varepsilon}\right)^{+}(x)}|\varphi(s)-\varphi(u(y))| d s\right) \psi^{0}\left(\vec{D}^{s} \overrightarrow{\bar{u}}_{\varepsilon}\right)\left|D_{x}^{j} \bar{u}_{\varepsilon}\right|\right] d y \\
& \leq 2 \varepsilon M_{n} \int_{\Omega}\left(\int_{u(y)-\varepsilon}^{u(y)+\varepsilon} \operatorname{Per}(\{\bar{u}(x) \geq \lambda\}) d \lambda\right) d y
\end{aligned}
$$

and we obtain that

$$
\lim _{\varepsilon \rightarrow 0^{+}} \frac{1}{\varepsilon} J_{2}^{n}=0 \quad \forall n \in \mathbb{N} .
$$

Then, by (4.30) and (4.31), we get

$$
\frac{1}{\varepsilon} I_{22}^{n} \geq o(\varepsilon) \quad \forall n \in \mathbb{N} .
$$

In a similar way, we also obtain that

$$
\frac{1}{\varepsilon} I_{23}^{n} \geq o(\varepsilon) \quad \forall n \in \mathbb{N} .
$$

Now, from (4.29), (4.32) and (4.33), we deduce that

$$
\frac{1}{\varepsilon} I_{2}^{n} \geq o(\varepsilon) \quad \forall n \in \mathbb{N} .
$$

Consequently, since

$$
\lim _{n \rightarrow \infty} \lim _{\varepsilon \rightarrow 0^{+}} \frac{1}{\varepsilon} I_{1}^{n}=\int_{\Omega}|u(x)-\bar{u}(x)| d x,
$$

and

$$
\lim _{n \rightarrow \infty} \lim _{\varepsilon \rightarrow 0^{+}} \frac{1}{\varepsilon} I_{3}^{n} \leq \int_{\Omega}|v(x)-\bar{v}(x)| d x,
$$

we deduce that

$$
\int_{\Omega}|u(x)-\bar{u}(x)| d x \leq \int_{\Omega}|v(x)-\bar{v}(x)| d x .
$$

In particular, this implies the uniqueness of entropy solutions of (4.25) when the right hand side $v \in L^{\infty}(\Omega)$.

Finally, assume that $v, \bar{v} \in L^{1}(\Omega)$ and $u, \bar{u} \in L^{1}(\Omega)$ are the corresponding entropy solutions of (4.25), and (4.26), respectively. Let $\bar{v}_{n} \in L^{\infty}(\Omega)$ be such 
that $\bar{v}_{n} \rightarrow v$ in $L^{1}(\Omega)$, and let $\bar{u}_{n}$ be the corresponding entropy solution. Observe that, from estimate (4.34) we deduce that

$$
\int_{\Omega}\left|\bar{u}(x)-\bar{u}_{n}(x)\right| d x \leq \int_{\Omega}\left|\bar{v}(x)-\bar{v}_{n}(x)\right| d x \rightarrow 0 .
$$

Hence $\bar{u}_{n} \rightarrow \bar{u}$. Again, from (4.34) we have

$$
\int_{\Omega}\left|u(x)-\bar{u}_{n}(x)\right| d x \leq \int_{\Omega}\left|v(x)-\bar{v}_{n}(x)\right| d x,
$$

and, letting $n \rightarrow \infty$ we obtain

$$
\int_{\Omega}|u(x)-\bar{u}(x)| d x \leq \int_{\Omega}|v(x)-\bar{v}(x)| d x .
$$

This concludes the proof of the Theorem.

REMARK 4.2. When applying Kruzhkov's method, if instead of multiplying by $T_{\varepsilon}(u(y)-\bar{u}(x))$ we multiply by $T_{\varepsilon}(u(y)-\bar{u}(x))^{+}$, we obtain the estimate

$$
\int_{\Omega}(u(x)-\bar{u}(x))^{+} d x \leq \int_{\Omega}(v(x)-\bar{v}(x))^{+} d x .
$$

REMARK 4.3. We observe that $\left(\mathrm{H}_{2}\right)$ is not used when $f(z, \xi)=\varphi(z) \psi(\xi)$ as in Remark 3.1. Indeed, it suffices to prove the analogous of (4.19). Let us comment on this in the case where $v \in L^{\infty}(\Omega)$, where we do not need to use truncatures. Since $\mathbf{a}(z, \xi)=\varphi(z) \mathbf{b}(\xi)$, if we denote by $J_{\varphi}(r):=\int_{0}^{r} \varphi(s) d s$, we have

$$
\begin{aligned}
\lim _{n \rightarrow \infty} \int_{\Omega} \phi \mathbf{a}\left(u_{n}, \nabla g\right) \cdot \nabla\left(u_{n}-g\right) d x & =\lim _{n \rightarrow \infty} \int_{\Omega} \phi \varphi\left(u_{n}\right) \mathbf{b}(\nabla g) \cdot \nabla\left(u_{n}-g\right) d x \\
& =\lim _{n \rightarrow \infty} \int_{\Omega} \phi \mathbf{b}(\nabla g) \cdot\left(\nabla J_{\varphi}\left(u_{n}\right)-\varphi\left(u_{n}\right) \nabla g\right) d x .
\end{aligned}
$$

Now, since $\nabla J_{\varphi}\left(u_{n}\right) \rightarrow D J_{\varphi}(u)$ weakly as measures, we have

$$
\lim _{n \rightarrow \infty} \int_{\Omega} \phi \mathbf{a}\left(u_{n}, \nabla g\right) \cdot \nabla\left(u_{n}-g\right) d x=\int_{\Omega} \phi\left[\mathbf{b}(\nabla g) \cdot D J_{\varphi}(u)-\varphi(u) \mathbf{b}(\nabla g) \cdot \nabla g\right]
$$

and (4.19) follows as in the proof above.

Remark 4.4. If $\mathbf{a}(z, \xi)=\frac{|z|^{5 / 2} \xi}{1+|z||\xi|}$ ([23], [14]), then Theorem 4.1 holds for any $v \in L^{\infty}(\Omega)$ such that $v \geq a$, for some $a>0$. In this case, we approximate $v$ by functions $v_{n} \in L^{\infty}(\Omega)$ such that $v_{n} \geq a$. Moreover, multiplying the equation

$$
u_{n}-a-\operatorname{div} \mathbf{a}_{n}\left(u_{n}, \nabla u_{n}\right)=v_{n}-a
$$

by $\left(u_{n}-a\right)^{-}=\min \left(u_{n}-a, 0\right)$ and integrating in $\Omega$ we obtain that

$$
\int_{\Omega}\left(\left(u_{n}-a\right)^{-}\right)^{2} d x \leq \int_{\Omega}\left(v_{n}-a\right)\left(u_{n}-a\right)^{-} d x \leq 0
$$

and we deduce that $u_{n} \geq a$. Hence $u \geq a$. 


\section{5. - Semigroup solution}

In this section we shall associate an $m$-accretive operator in $L^{1}(\Omega)$ to the formal differential expression $-\operatorname{div} \mathbf{a}(u, \nabla u)$ together with the Neumann boundary conditions.

Definition 5.1. $(u, v) \in \mathcal{B}_{0}$ if and only if $u \in T B V(\Omega), v \in L^{1}(\Omega)$ and $u$ is an entropy solution of problem (3.1), that is, $\mathbf{a}(u, \nabla u) \in X_{1}(\Omega)$ and satisfies:

$$
\begin{gathered}
v=-\operatorname{div} \mathbf{a}(u, \nabla u) \quad \text { in } \quad \mathcal{D}^{\prime}(\Omega) \\
\mathbf{a}(u, \nabla u) \cdot D^{s} T_{a, b}(u) \geq\left(\mathcal{R}_{f}^{a, b}\right)^{s}(u) \quad \forall a<b, \\
{[\mathbf{a}(u, \nabla u), v]=0 \quad \mathcal{H}^{N-1}-\text { a.e. } \text { on } \partial \Omega .}
\end{gathered}
$$

Let us define the operator $B=\mathcal{B}_{0} \cap\left(L^{\infty}(\Omega) \times L^{\infty}(\Omega)\right)$.

Let $(u, v) \in \mathcal{B}_{0}, w \in B V(\Omega) \cap L^{\infty}(\Omega)$, and $T=T_{a, b}, a<b$. Multiplying (5.1) by $w-T(u)$, using Green's formula (2.17) and having in mind that (5.2) is equivalent to (3.13), we obtain

$$
\begin{aligned}
\int_{\Omega}(w-T(u)) v d x= & -\int_{\Omega}(w-T(u)) \operatorname{div} \mathbf{a}(u, \nabla u) d x \\
= & \int_{\Omega}(\mathbf{a}(u, \nabla u), D w-D T(u)) \\
& -\int_{\partial \Omega}[\mathbf{a}(u, \nabla u), v](w-T(u)) d \mathcal{H}^{N-1} \\
\leq & \int_{\Omega}(\mathbf{a}(u, \nabla u), D w)-\mathcal{R}_{h}^{a, b}(u) .
\end{aligned}
$$

Therefore, if $(u, v) \in \mathcal{B}_{0}$, we have that

$$
\int_{\Omega}(w-T(u)) v d x \leq \int_{\Omega}(\mathbf{a}(u, \nabla u), D w)-\mathcal{R}_{h}^{a, b}(u),
$$

for all $w \in B V(\Omega) \cap L^{\infty}(\Omega)$.

PROPOSITION 5.2. Assume we are under assumptions $(H)$. Then $B$ is accretive in $L^{1}(\Omega), L^{\infty}(\Omega) \subseteq R(I+B)$ and $D(B)$ is dense in $L^{1}(\Omega)$. Moreover, if we assume that (3.5) holds for any $(z, \xi) \in \mathbb{R} \times \mathbb{R}^{N}$, then $\mathcal{B}_{0}$ is accretive in $L^{1}(\Omega)$, and $R\left(I+\mathcal{B}_{0}\right)=L^{1}(\Omega)$. 
Again, we observe that assumption $\left(\mathrm{H}_{2}\right)$ is not required if $f(z, \xi)=$ $\varphi(z) \psi(\xi)$, being $\varphi$ a bounded $C^{1}$ function such that $\varphi(z) \geq \alpha_{R}>0$ for all $z \in \mathbb{R},|z| \leq R$ (see Remark 3.1).

PROOF. The accretivity of the operator $\mathcal{B}_{0}$ in $L^{1}(\Omega)$ and the range condition follow from Theorem 4.1.

To prove the density of $D(B)$ in $L^{1}(\Omega)$, we prove that $C_{0}^{\infty}(\Omega) \subseteq \overline{D(B)}{ }^{L^{1}(\Omega)}$. Let $v \in C_{0}^{\infty}(\Omega)$. By Theorem 4.1, $v \in R\left(I+\frac{1}{n} B\right)$ for all $n \in \mathbb{N}$. Thus, for each $n \in \mathbb{N}$, there exists $u_{n} \in D(B),\left\|u_{n}\right\|_{\infty} \leq\|v\|_{\infty}$, such that $\left(u_{n}, n\left(v-u_{n}\right)\right) \in B$. Consequently, using $T=T_{\|v\|_{\infty}}$ in (5.4), we get

$$
\int_{\Omega}\left(w-u_{n}\right) n\left(v-u_{n}\right) d x \leq \int_{\Omega}\left(\mathbf{a}\left(u_{n}, \nabla u_{n}\right), D w\right)-\mathcal{R}_{h}\left(u_{n}\right),
$$

for all $w \in B V(\Omega) \cap L^{\infty}(\Omega)$. Taking $w=v$, we get

$$
\begin{aligned}
\int_{\Omega}\left(v-u_{n}\right)^{2} d x & \leq \frac{1}{n}\left(\int_{\Omega} \mathbf{a}\left(u_{n}, \nabla u_{n}\right) \cdot \nabla v d x-\mathcal{R}_{h}\left(u_{n}\right)\right) \\
& \leq \frac{1}{n} \int_{\Omega} \mathbf{a}\left(u_{n}, \nabla u_{n}\right) \cdot \nabla v d x \leq \frac{M}{n} \int_{\Omega}|\nabla v| d x .
\end{aligned}
$$

Letting $n \rightarrow \infty$, it follows that $u_{n} \rightarrow v$ in $L^{2}(\Omega)$, hence in $L^{1}(\Omega)$. Therefore $v \in \overline{D(B)}^{L^{2}(\Omega)} \subset \overline{D(B)}^{L^{1}(\Omega)}$ and the proof is complete.

From Proposition 5.2, if we denote by $\mathcal{B}$ the closure in $L^{1}(\Omega)$ of the operator $B$, it follows that $\mathcal{B}$ is m-accretive in $L^{1}(\Omega)$ and $\overline{D(\mathcal{B})}{ }^{L^{1}(\Omega)}=L^{1}(\Omega)$. Observe that, in case that (3.5) holds for any $(z, \xi) \in \mathbb{R} \times \mathbb{R}^{N}$, then $\mathcal{B}_{0}$ is a closed operator, since it is $m$-accretive (i.e., it is accretive and satisfies the range condition). Hence $\mathcal{B} \subseteq \mathcal{B}_{0}$, and being both operators $m$-accretive in $L^{1}(\Omega)$, then $\mathcal{B}=\mathcal{B}_{0}$. Thus, from now on we shall write both operators as $\mathcal{B}$. From Proposition 5.2, and according to the general theory of nonlinear semigroups (c.f., e.g., [11]), for any $u_{0} \in L^{1}(\Omega)$ there exists a unique mild solution $u \in C\left([0, T] ; L^{1}(\Omega)\right)$ of the abstract Cauchy problem

$$
u^{\prime}(t)+\mathcal{B} u(t) \ni 0, \quad u(0)=u_{0} .
$$

Moreover, $u(t)=T(t) u_{0}$ for all $t \geq 0$, being $(T(t))_{t \geq 0}$ the semigroup in $L^{1}(\Omega)$ generated by the Crandall-Liggett's exponential formula, i.e.,

$$
T(t) u_{0}=\lim _{n \rightarrow \infty}\left(I+\frac{t}{n} \mathcal{B}\right)^{-n} u_{0}
$$

Lemma 5.3. Given $\lambda>0$, and $u \in L^{q}(\Omega), 1 \leq q \leq \infty$, if $v=(I+\lambda \mathcal{B})^{-1} u$, then

$$
\|v\|_{q} \leq\|u\|_{q}
$$


As a consequence, for any $u_{0} \in L^{q}(\Omega), 1 \leq q \leq \infty$, we have

$$
T(t) u_{0} \in L^{q}(\Omega) \quad \forall t \geq 0 .
$$

Proof. Let us prove the assertion assuming that $u \in L^{\infty}(\Omega)$. In that case we know that $v=(I+\lambda \mathcal{B})^{-1} u=(I+\lambda B)^{-1} u \in L^{\infty}(\Omega)$. Since $v=(I+\lambda B)^{-1} u$, we have $\left(v, \frac{1}{\lambda}(u-v)\right) \in B$. Then, $\mathbf{a}(v, \nabla v) \in X_{1}(\Omega)$ and the following holds:

$$
\begin{gathered}
\frac{1}{\lambda}(u-v)=-\operatorname{div} \mathbf{a}(v, \nabla v) \quad \text { in } \quad \mathcal{D}^{\prime}(\Omega), \\
\mathbf{a}(v, \nabla v) \cdot D^{s} v \geq\left(\mathcal{R}_{f}\right)^{s}(v), \\
{[\mathbf{a}(v, \nabla v), v]=0 \quad \mathcal{H}^{N-1}-\text { a.e. on } \partial \Omega .}
\end{gathered}
$$

Let $\mathcal{P}_{0}$ be the set of functions defined in the proof of Theorem 4.1. For $p \in \mathcal{P}_{0}$, by Green's formula, we have

$$
\begin{aligned}
\int_{\Omega} p(v)(v-u) d x & =\lambda \int_{\Omega} p(v) \operatorname{div} \mathbf{a}(v, \nabla v) d x=-\lambda \int_{\Omega}(\mathbf{a}(v, \nabla v), D p(v)) \\
& =-\lambda \int_{\Omega} p^{\prime}(v) \mathbf{a}(v, \nabla v) \cdot \nabla v d x-\lambda \int_{\Omega} \mathbf{a}(v, \nabla v) \cdot D^{s}(p(v)) .
\end{aligned}
$$

Now, by (3.6), it follows that

$$
\int_{\Omega} p^{\prime}(v) \mathbf{a}(v, \nabla v) \cdot \nabla v d x \geq 0 .
$$

On the other hand, by the chain rule for BV-functions (see [1]), we have $D^{s}(p(v))=\bar{p}_{v} D^{s} v$ with $\bar{p}_{v} \geq 0, \bar{p}_{v}$ being the Vol'pert averaged superposition. Moreover, by [7],

$$
\theta(\mathbf{a}(v, \nabla v), D p(v), \cdot)=\theta(\mathbf{a}(v, \nabla v), D v, \cdot) \quad|D v|-\text { a.e. }
$$

Then,

$$
\begin{aligned}
\mathbf{a}(v, \nabla v) \cdot D^{s}(p(v)) & =\theta(\mathbf{a}(v, \nabla v), D p(v), \cdot)\left|D^{s} p(v)\right| \\
& =\bar{p}_{v} \theta(\mathbf{a}(v, \nabla v), D v, \cdot)\left|D^{s} v\right| \\
& =\bar{p}_{v} \mathbf{a}(v, \nabla v) \cdot D^{s} v \geq \bar{p}_{v}\left(\mathcal{R}_{f}\right)^{s}(v) \geq 0 .
\end{aligned}
$$

Therefore, we get

$$
\int_{\Omega} p(v)(v-u) d x \leq 0
$$

and consequently,

$$
\int_{\Omega} p(v) v d x \leq \int_{\Omega} p(v) u d x \quad \forall p \in \mathcal{P}_{0} .
$$

This implies (5.6), having in mind a result of [10].

Finally, let us consider the case where $u \in L^{q}(\Omega), v=(I+\lambda \mathcal{B})^{-1} u$. Let $u_{n} \in L^{\infty}(\Omega)$ be such that $u_{n} \rightarrow u$ in $L^{q}(\Omega)$. Then, if $v_{n}=(I+\lambda B)^{-1} u_{n}$, we have that $\left\|v_{n}\right\|_{q} \leq\left\|u_{n}\right\|_{q}$, and $v_{n} \rightarrow v$ in $L^{1}(\Omega)$. Hence, letting $n \rightarrow \infty$ we obtain $\|v\|_{q} \leq\|u\|_{q}$.

Last assertion is a consequence of (5.6).

In [6] we shall define the notion of entropy solution for (1.2), and we shall prove that entropy solutions are unique and coincide with semigroup solutions. 


\section{REFERENCES}

[1] L. Ambrosio - N. Fusco - D. Pallara, "Functions of Bounded Variation and Free Discontinuity Problems", Oxford Mathematical Monographs, The Clarendom Press, Oxford University Press, 2000.

[2] F. Andreu - V. Caselles - J.M. Mazón, A parabolic quasilinear problem for linear growth functionals, Rev. Mat. Iberoamericana 18 (2002), 135-185.

[3] F. ANdReu - V. CASElles - J.M. Mazón, Existence and uniqueness of solution for a parabolic quasilinear problem for linear growth functionals with $L^{1}$ data, Math. Ann. 322 (2002), 139-206.

[4] F. Andreu - V. Caselles - J.M. Mazón, The Cauchy Problem for Linear Growth Functionals with, J. Evol. Equ. 3 (2003), 39-65.

[5] F. Andreu - V. CASelles - J.M. Mazón, "Parabolic Quasilinear Equations Minimizing Linear Growth Functionals", Progress in Mathematics, vol. 223, Birkhäuser-Verlag, Basel, 2004.

[6] F. Andreu - V. CAselles - J.M. Mazón, A Strongly Degenerate Quasilinear Equation: the Parabolic Case, preprint, 2003.

[7] G. Anzellotti, Pairings Between Measures and Bounded Functions and Compensated Compactness, Ann. Mat. Pura Appl. (4) 135 (1983), 293-318.

[8] G.I. Barenblatt - M. Bertsch - R. Dal Passo - V.M. Prostokishin - M. Ughi, A mathematical Model of Turbulent Heat and Mass Transfer in Stable Stratified Shear Flow, J. Fluid Mech. 253 (1993), 341-358.

[9] Ph. Bénilan - L. Boccardo - T. Gallouet - R. Gariepy - M. Pierre - J.L Vazquez, An $L^{1}$-Theory of Existence and Uniqueness of Solutions of Nonlinear Elliptic Equations, Ann. Scuola Norm. Sup. Pisa Cl. Sci. (4) 22 (1995), 241-273.

[10] Ph. BÉnilan - M.G. Crandall, Completely Accretive Operators, In: "Semigroups Theory and Evolution Equations", Ph. Clement et al. (eds.), Marcel Dekker, 1991, pp. 41-76.

[11] Ph. Bénilan - M.G. Crandall - A. Pazy, "Evolution Equations Governed by Accretive Operators", Book in preparation.

[12] M. Bertsch - R. Dal Passo, Hyperbolic Phenomena in a Strongly Degenerate Parabolic Equation, Arch. Ration. Mech. Anal. 117 (1992), 349-387.

[13] M. Bertsch - R. DAl PAsso, A Parabolic Equation with Mean-Curvature Type Operator, In: "Progres Nonlinear Differential Equation Appl.”, F. Browder (ed.), 7 Birkhäuser, 1992, 89-97.

[14] Рн. Blanc, On the regularity of the solutions of some degenerate parabolic equations, Comm. Partial Differential Equations 18 (1993), 821-846.

[15] $\mathrm{PH}$. Blanc, Sur une classe d'equations paraboliques degeneréesa une dimension d'espace possedant des solutions discontinues, Ph.D. Thesis, number 798, Ecole Polytechnique Federale de Lausanne, 1989.

[16] D. Blanchard - F. Murat, Renormalized solutions on nonlinear parabolic problems with $L^{1}$ data: existence and uniqueness, Proc. Royal Soc. Edinburgh Sect. A 127 (1997), 1137-1152.

[17] J. CARRILlo - P. Wittbold, Uniqueness of Renormalized Solutions of Degenerate EllipticParabolic problems, J. Differential Equations 156 (1999), 93-121. 
[18] M. G. Crandall, Nonlinear Semigroups and Evolution Governed by Accretive Operators, In: "Proceeding of Symposium in Pure Mat.", Part I, F. Browder (ed.) A.M.S., Providence 1986, 305-338.

[19] M. G. CRAndall - T. M. Liggett, Generation of Semigroups of Nonlinear Transformations on General Banach Spaces, Amer. J. Math. 93 (1971), 265-298.

[20] G. DaL Maso, Integral representation on $B V(\Omega)$ of $\Gamma$-limits of variational integrals, Manuscripta Math. 30 (1980), 387-416.

[21] R. Dal Passo, Uniqueness of the entropy solution of a strongly degenerate parabolic equation, Comm. Partial Differential Equations 18 (1993), 265-279.

[22] E. De Giorgi - L. Ambrosio, Un nuovo tipo di funzionale del calcolo delle variazioni, Atti Accad. Naz. Lincei Cl. Sci. Fis. Mat. Natur., Rend. Lincei (9) Mat. Appl., (8) 82 (1988), 199-210.

[23] J. J. Duderstadt - G. A. Moses, "Inertial Confinement Fusion", John Wiley \& Sons, 1982.

[24] L. C. Evans - R. F. Gariepy, "Measure Theory and Fine Properties of Functions", Studies in Advanced Math., CRC Press, 1992.

[25] M. A. Krasnosel'skit - Ya. B. Rutickit, "Convex Functions and Orliz Spaces”, P. Noordholff, Groningen, 1961.

[26] R. Kонn - R. Tемам, Dual space of stress and strains with application to Hencky plasticity, Appl. Math. Optim. 10 (1983), 1-35.

[27] S. N. KRUZhKov, First order quasilinear equations in several independent variables, Math. USSR-Sb. 10 (1970), 217-243.

[28] J. LERAY - J. L. LiONS, Quelques resultats de Visik sur les problemes elliptiques semilineaires par les metode de Minty et Browder, Bull. Soc. Math. France 93 (1965), 97-107.

[29] P. Rosenau, Free Energy Functionals at the High Gradient Limit, Phys. Rev. A 41 (1990), 2227-2230.

[30] W.P. ZiEMER, “Weakly Differentiable Functions”, GTM 120, Springer Verlag, 1989.

Universitat de Valencia

Dept. de Análisis Matemático

Universitat Pompeu-Fabra

Dept. de Tecnologia

Universitat de Valencia

Dept. de Análisis Matemático 